ALEA, Lat. Am. J. Probab. Math. Stat. 13, 239-264 (2016)

DOI: $10.30757 /$ ALEA.v13-10

\title{
A third-moment theorem and precise asymptotics for variations of stationary Gaussian sequences
}

\section{Léo Neufcourt and Frederi G. Viens}

Department of Statistics, Columbia University. 1255 Amsterdam Avenue, Room 1005 SSW, MC 4690. New York, NY, USA; and ex-affiliate of CMAP, Ecole Polytechnique. Route de Saclay, UMR CNRS 7641. Palaiseau, France.

E-mail address: ln2294@columbia.edu

Department of Statistics, Purdue University. 150 N. University St., Office MATH 200. West Lafayette, IN, USA. Phone: (765)494-6035. Fax: (765)494-0558.

E-mail address: viens@stat.purdue.edu

URL: http://www.stat.purdue.edu/ viens/

\begin{abstract}
In two new papers Biermé et al. (2012) and Nourdin and Peccati (2015), sharp general quantitative bounds are given to complement the well-known fourth moment theorem of Nualart and Peccati, by which a sequence in a fixed Wiener chaos converges to a normal law if and only if its fourth cumulant converges to 0 . The bounds show that the speed of convergence is precisely of order the maximum of the fourth cumulant and the absolute value of the third moment (cumulant). Specializing to the case of normalized centered quadratic variations for stationary Gaussian sequences, we show that a third moment theorem holds: convergence occurs if and only if the sequence's third moments tend to 0 . This is proved for sequences with general decreasing covariance, by using the result of Nourdin and Peccati (2015), and finding the exact speed of convergence to 0 of the quadratic variation's third and fourth cumulants. Nourdin and Peccati (2015) also allows us to derive quantitative estimates for the speeds of convergence in a class of logmodulated covariance structures, which puts in perspective the notion of critical Hurst parameter when studying the convergence of fractional Brownian motion's quadratic variation. We also study the speed of convergence when the limit is not Gaussian but rather a second-Wiener-chaos law. Using a log-modulated class of spectral densities, we recover a classical result of Dobrushin-Major/Taqqu whereby the limit is a Rosenblatt law, and we provide new convergence speeds. The conclusion in this case is that the price to pay to obtain a Rosenblatt limit despite a slowly varying modulation is a very slow convergence speed, roughly of the same order as the modulation.
\end{abstract}

Received by the editors October 3, 2015; accepted January 6, 2016.

2010 Mathematics Subject Classification. 60G15, 60F05, 60H07, 60G22.

Key words and phrases. Stationary Gaussian Process, Wiener Space, Central Limit Theorem, Berry-Esséen, Breuer-Major, Second Chaos. 


\section{Introduction}

We are inspired by the following reformulation of Theorem 1.2 in Nourdin and Peccati (2015), which is itself based on ideas contained in Biermé et al. (2012).

Theorem 1.1 ( $4^{\text {th }}$ moment theorem in total variation and convergence rates ). If $\left(F_{n}\right)_{n \geq 0}$ is a sequence in a fixed Wiener chaos (e.g. in the second chaos), and $\operatorname{Var}\left[F_{n}\right]=1$, then $\left(F_{n}\right)_{n \geq 0}$ converges in law towards $\mathcal{N}(0,1)$ if and only if $\mathbf{E}\left[F_{n}^{4}\right] \rightarrow$ $3=\mathbf{E}\left[N^{4}\right]$, where $N \sim \mathcal{N}(0,1)$. Moreover the convergence rate in this case is $M_{n}:=\max \left(\mathbf{E}\left[F_{n}^{4}\right]-3,\left|\mathbf{E}\left[F_{n}^{3}\right]\right|\right)$, in the sense of commensurability for the total variation metric $d_{T V}\left(F_{n}, N\right) \asymp M_{n}$, i.e.

$$
\exists c, C>0: c M_{n} \leq d_{T V}\left(F_{n}, N\right) \leq C M_{n} .
$$

The first part of this theorem is known as the 4th moment theorem, proved originally by Nualart and Peccati in Nualart and Peccati (2005). The second part, i.e. relation (1.1), suggests that the third moment is just as important as the 4th moment when investigating the normal convergence of sequences in a fixed Wiener chaos. Theorem 1.1 also provides new information about a rather successful estimate for evaluating normal convergence speeds, which was established thanks to a research program started in 2008 by Nourdin and Peccati in Nourdin and Peccati (2009). Specifically, in Nourdin et al. (2010) an upper bound on $d_{T V}\left(F_{n}, N\right)$ of the form $C \sqrt{\mathbf{E}\left[F_{n}^{4}\right]-3}$ is established (see Theorem 5.2.6 in Nourdin and Peccati, 2012); Theorem 1.1 above shows that this estimate is not sharp in cases where the third moment is dominated by the 4 th moment minus 3 (a.k.a. the 4 th cumulant $\kappa_{4}\left(F_{n}\right)$ ), and in the other cases, leaves the question of sharpness of past results to a comparison with the third moment. Such a discovery begs the question of how much one might improve certain convergence results, e.g. of Berry-Esséen type, by using Theorem 1.1 instead of Theorem 5.2.6 in Nourdin and Peccati (2012). The authors of Nourdin and Peccati (2012) joined forces with Biermé and Bonami to produce the first positive result in this direction, in Biermé et al. (2012): they worked with a weaker notion of convergence than total-variation convergence, but were able to show, in the case of the power variations of discrete fractional Brownian motion, that the third moment seems to dominate the 4th cumulant in many cases, and therefore determines normal convergence in those cases, yielding much improved speeds as a consequence. In Nourdin and Peccati (2015), Nourdin and Peccati improve this result by showing that it holds for the total variation distance, thanks to the general Theorem 1.1.

Herein, we too base our analysis on the power afforded by Theorem 1.1, and focus our attention on broadening the study of quadratic variations from Biermé et al. (2012), to include general stationary Gaussian sequences with no reference to Hölder-continuity or self-similarity.

In addition to what is described above, our motivation is to show that, in the case of quadratic variations (which live in the 2 nd chaos) the fourth moment theorem can be replaced by a third moment theorem, with corresponding quantitative estimates of the total variation distance and of the relation between the 3rd and 4th moments. Another motivation is to keep our analysis as general as possible within the confines of variations of stationary Gaussian sequences, with as little assumptions about their covariance structure as we can.

In other words, we consider a sequence of centered identically distributed Gaussian random variables $\left(X_{n}\right)_{n \in \mathbf{Z}}$ for which there exists a function $\rho$ on $\mathbf{Z}$ such that 
for all integers $k, n, \mathbf{E}\left[X_{n} X_{n+k}\right]=\rho(k)$. We only assume that $\rho$ is of constant sign, and $|\rho|$ decreases near $+\infty$. Note that $\rho$ is necessarily symmetric (even) and of positive type (meaning that $(\rho(k-\ell))_{k, \ell}$ is a non-negative definite matrix). Without loss of generality, we assume that $\operatorname{Var}\left[X_{n}\right]=\rho(0)=1$ throughout. We define the normalized centered quadratic variation

$$
F_{n}:=\frac{V_{n}}{\sqrt{v_{n}}}
$$

where $V_{n}:=\frac{1}{\sqrt{n}} \sum_{k=0}^{n-1}\left(X_{k}^{2}-1\right)$, and $v_{n}:=E\left[V_{n}^{2}\right]$. We prove the equivalence of third and fourth moment theorems for the limit of $F_{n}$ under this general framework (Theorem 3.2 on page 246).

The presence of the "normalizing" term $1 / \sqrt{n}$ in the definition of $V_{n}$ is not needed (Theorem 3.2 remains valid without it); it is a convention that comes from working with sequences where $v_{n}$ is bounded, so that the normalization of the discrete centered quadratic variation $\sum_{k=0}^{n-1}\left(X_{k}^{2}-1\right)$ needed to obtain a normal limit is $1 / \sqrt{n}$, as one would hope for in a straightforward generalization of the central limit theorem. This situation is the well-known framework for the classical Breuer-Major central limit theorem (see Breuer and Major, 2011 or Theorem 7.2.4 in Nourdin and Peccati, 2012). Some might argue that the interesting cases are those for which $v_{n}$ tends to infinity. See step 2 in the proof of Proposition 4.1 in Section 4 for a class of log-modulated models where the transition from bounded to unbounded $v_{n}$ occurs, i.e. where the Breuer-Major statement becomes invalid. Generally speaking, we argue that this type of "phase transition" is an artefact of the model class one uses.

We investigate this question of phase transitions in the Berry-Esséen rates for the Breuer-Major central limit theorem, i.e. the rate at which $d_{T V}\left(F_{n}, N\right)$ converges to 0 . In the case of fractional Gaussian noise (fGn, for which $\rho(k)$ is equivalent to $H(2 H-1)|k|^{2 H-2}$ for large $\left.|k|\right)$, up until very recently, the rate of convergence of $d_{T V}\left(F_{n}, N\right)$ to 0 was known to be the classical Berry-Esséen rate $1 / \sqrt{n}$ only for $H<5 / 8$. Thanks to the results in Nourdin and Peccati (2015) and Biermé et al. (2012), it is now known that this rate hold up to $H<2 / 3$. The optimal rates for fBm are even known, as can be seen in Biermé et al. (2012) (also see Proposition 4.3 in Nourdin and Peccati (2015)): the rate $1 / \sqrt{n}$ is optimal for $H<2 / 3$; then for $H=2 / 3$, the optimal rate is $n^{-1 / 2} \log ^{2} n$, whereas for $H \in(2 / 3,3 / 4)$, the optimal rate is $n^{6 H-9 / 2}$. Therefore, speaking strictly about the fGn scale, this can be interpreted as a "phase transition", or a critical threshold $H=2 / 3$. We argue that for general sequences, insofar as optimal rates are given by (1.1) in Theorem 1.1 if they can be computed, the notion of critical threshold is model-class-dependent.

Herein, we show that the so-called critical threshold above can be investigated in more detail, to reveal a range of possibilities for the convergence rate at the Berry-Esséen critical threshold $H=2 / 3$, for a class of covariance functions with log-modulation. Our tools also enable us to compute rates for situations which were left out in Nourdin and Peccati (2015) and Biermé et al. (2012): a second critical threshold at $H=3 / 4$ reveals another range of possibilities under our log-modulated class where normal convergence holds. This subclass of models with " $H=3 / 4$ " contains models for which the Breuer-Major theorem holds, and others for which it does not. When normal convergence holds but the classical Breuer-Major normalization fails, we find very slow rates of convergence: rate $\log ^{-\frac{3}{2}}(n)$ in all cases 
except one exceptional model which benefits from a $\log (\log (n))^{-\frac{3}{2}}$ correction. For fGn, which is a special case of our results, we provide a slight improvement on the only result we are aware of (Corollary 7.4.3 in Nourdin and Peccati, 2012) which deals with the case " $H=3 / 4$ ": scaled quadratic variations for fGn with $H=3 / 4$ have a speed of normal convergence of order $\log ^{-1}(n)$. All our results mentioned above are sharp, and are given in Proposition 4.1 on page 249.

Both this class of examples, given in Section 4, and our generic third moment theorem given in Section 3, are based on a precise asymptotic expression for the third moment of $F_{n}$, and a precise upper bound for the fourth moment of $F_{n}$, both given for general $\rho$, in Theorem 2.4 in Section 2. This intrinsic study shows that there is nothing special about the power rate of correlation decay when investigating central limit theorems for quadratic variations. We also note that we are able to develop a general third-moment theorem without needing a direct estimation of the fourth cumulant $\kappa_{4}\left(F_{n}\right)$, giving more credence to the claim that the third moment theorem is the right tool for studying centered quadratic variations.

The key to this surprising shortcut is Proposition 3.1, which shows that one can bound the third moment $\kappa_{3}\left(F_{n}\right)$ below by $n^{1 / 4} \kappa_{4}\left(F_{n}\right)^{3 / 4}$. This implies that $\kappa_{3}\left(F_{n}\right)$ is always the dominant term in the exact rate of convergence of $d_{T V}\left(F_{n}, N\right)$ to zero, showing in particular that if $\kappa_{3}\left(F_{n}\right) \rightarrow 0$ then $\kappa_{4}\left(F_{n}\right) \rightarrow 0$, which is sufficient to conclude that $F_{n} \rightarrow N$. This in turn implies the exact total-variation convergence rate (1.1) from Theorem 1.1. The converse statement, that $\kappa_{4}\left(F_{n}\right) \rightarrow 0$ implies $\kappa_{3}\left(F_{n}\right) \rightarrow 0$, follows immediately from Theorem 1.1.

The remainder of the paper after Section 4 deals with what happens when no normal convergence holds, i.e. when none of the equivalent conditions in Theorem 3.2 hold. Here, as is typical for studies of non-normal convergence on Wiener space, the Malliavin calculus-based tools such as Theorem 1.1 are no longer useful. In such cases one may expect that normalized centered quadratic variations of stationary Gaussian sequences will converge in distribution towards second-chaos laws.

The classical result in this direction is a special case of what is often known as the Dobrushin-Major-Taqqu theorem. This theorem refers to two separate results from 1979 on non-linear functionals of fractional Brownian motion (fBm). In the special case of centered quadratic variations, Taqqu (1979) implies a convergence in the mean-square for the normalized centered quadratic variations of fBm on $[0,1]$, towards the law of a Rosenblatt r.v. $F_{\infty}$. On the other hand Dobrushin and Major (1979) prove convergence to the same law, but only in law, not in the mean-square, for the increments of $\mathrm{fBm}$ over intervals of length 1 , as the horizon increases. This second setting is the same as the one we use here: the increments of $\mathrm{fBm}$ on unit intervals are known as fractional Gaussian noise (fGn), and as mentioned above, fGn is the canonical power-scale example of a stationary Gaussian sequence with unit variance. The self-similarity of fBm implies that Taqqu's result and Dobrushin and Major's result are equivalent in the setting we have chosen here, i.e. stationary Gaussian sequences. For the historical reasons given above, it is legitimate to refer to such a result as a Dobrushin-Major theorem.

Herein, we investigate the question of speed of convergence in the DobrushinMajor theorem. As we said, results of the type of Theorem 1.1 are of no use here, since the convergence we seek is of second-chaos type, not normal. Instead, as in Breton and Nourdin (2008), we refer to a result of Davydov and Martynova (1989), which enables one to compare the total-variation distance of two chaos variables 
with the square-root of their difference's $L^{2}$ norm. This requires the use of a change of probability space, which is why the convergence cannot hold in $L^{2}(\Omega)$. In the spirit of Dobrushin and Major, we consider the spectral representation of the stationary Gaussian sequence, and obtain first a general strategy for estimating the speed of convergence for sequences whose spectral density has a certain type of functional asymptotic behavior under dilation of the Fourier space's unit circle (Section 5). Then we specialize to cases where the spectral density is a power times a slowly-varying function, in order to present results similar to those for our logmodulated class of examples. Our computational technique must go beyond the calculations in Breton and Nourdin (2008), since the limit we obtain is the same for all log-modulated processes for a fixed $H$, and therefore no self-similarity arguments may be used. Our results in Section 6 show that the speed of convergence itself does not depend on the modulation parameter: for modulations of the form $\log ^{\beta}|x|$, $d_{T V}\left(F_{n}, F_{\infty}\right)=\mathcal{O}\left(\log ^{-2} n\right)$, for any $\beta>0$ (Theorem 2 on page 256$)$.

We do not know if this result is sharp, but it does provide a sobering extension to known speed-of-convergence results in the Dobrushin-Major theorem, as compared for instance with the case $\beta=0$, where we get the much faster power rate $d_{T V}\left(F_{n}, F_{\infty}\right)=\mathcal{O}\left(n^{3 / 4-H}\right)$ (see Theorem 7.4.5 in Nourdin and Peccati, 2012). One can interpret our result as saying that the price to pay for a mild "universality" result whereby a slowly-varying perturbation of fGn still leads to a Rosenblatt limit in law, is that the speed of convergence will be roughly as slow as the perturbation.

Summarizing the descriptions above, the remainder of this paper is structured as follows. In Section 2 we find sharp estimates of the variance, third, and fourth cumulants of the normalized centered quadratic variation $F_{n}$. We use these in Section 3 to prove a third-moment theorem for normal convergence, with precise speed of convergence, for general stationary Gaussian sequences (Theorem 3.2). In Section 4 , we apply this theorem to a class of log-modulated covariance structures, identifying a number of critical cases in the convergence rates (Proposition 4.1), which go beyond the phase transitions recently identified for fGn in Biermé et al. (2012). In Section 5, we define a strategy for estimating speeds of non-normal convergence for general $F_{n}$, based on a classical estimate in Davydov and Martynova (1989) and spectral representation of stationary sequences (Corollary 1). In Section 6, we apply this strategy for sequences with power spectral density and log modulation, proving that compared to the fGn case with $H>3 / 4$, the speed deteriorates to $\log ^{-2} n$ instead of $n^{3 / 4-H}$ (Theorem 2).

\section{Estimates for the second, third, and fourth cumulants}

In this section we get precise estimates for the variance and the $3^{\text {rd }}$ and $4^{\text {th }}$ cumulants of $F_{n}$. Let us define first the comparison relations that are used in what follows.

Definition 2.1 (Comparison relations). Given two deterministic numeric sequences $\left(a_{n}\right)_{n \geq 0},\left(b_{n}\right)_{n \geq 0}$ in a metric space, we use the following notations and definitions for respectively domination, commensurability, equivalence:

$a_{n}=\mathcal{O}\left(b_{n}\right) \Longleftrightarrow \exists C>0: a_{n} \leq C b_{n}$ for $n$ large enough

$a_{n} \asymp b_{n} \Longleftrightarrow \exists c, C>0: c b_{n} \leq a_{n} \leq C b_{n}$ for $n$ large enough

$a_{n} \sim b_{n} \Longleftrightarrow \exists c_{n}, C_{n}>0: \lim _{n \rightarrow \infty} c_{n}=\lim _{n \rightarrow \infty} C_{n}=1$ and $c_{n} b_{n} \leq a_{n} \leq C_{n} b_{n}$ for $n$ large enough. 
Remark 2.2. $\mathcal{O}$ is an order relation, while $\asymp$ and $\sim$ are equivalence relations. Moreover, $a_{n} \asymp b_{n}$ is equivalent to $\left\{a_{n}=\mathcal{O}\left(b_{n}\right)\right.$ and $\left.b_{n}=\mathcal{O}\left(a_{n}\right)\right\}$.

Remark 2.3. The quantities $\kappa_{3}\left(F_{n}\right):=E\left(F_{n}^{3}\right)$ and $\kappa_{4}\left(F_{n}\right):=\mathbf{E}\left[F_{n}^{4}\right]-3$ are called the 3rd and 4th cumulants of $F_{n}$. That $\kappa_{3}\left(F_{n}\right)$ coincides with the third moment is because $F_{n}$ is centered. Moreover, $\kappa_{4}\left(F_{n}\right)$ is strictly positive because $F_{n}$ is a non-Gaussian chaos r.v. (see Nourdin and Peccati, 2012, Appendix A for details on cumulants on Wiener chaos).

Theorem 2.4. Let $\left(F_{n}\right)_{n \geq 0}$ be the sequence of normalized centered quadratic variations of a centered stationary Gaussian sequence $\left(X_{n}\right)_{n \in \mathbf{Z}}$ with covariance $\rho$, i.e. with $\mathbf{E}\left[X_{n} X_{n+k}\right]=\rho(k)$ for $n, k \in \mathbf{Z}$, we let $F_{n}:=V_{n} / \sqrt{v_{n}}$, where $V_{n}:=$ $n^{-1 / 2} \sum_{k=0}^{n-1}\left(X_{k}^{2}-1\right)$, and $v_{n}:=E\left[V_{n}^{2}\right]$. Let $\kappa_{3}\left(F_{n}\right)$ and $\kappa_{4}\left(F_{n}\right)$ be defined in Remark 2.3. Assume that the sequence of correlations $\rho$ has a constant sign, and that $|\rho|$ is decreasing near $+\infty$, and $\rho(0)=1$. Then for large $n$,

$$
\begin{aligned}
\frac{1}{4} \frac{8}{v_{n}^{3 / 2} \sqrt{n}}\left(\sum_{|k|<n}|\rho(k)|^{3 / 2}\right)^{2} & \leq\left|\kappa_{3}\left(F_{n}\right)\right| \leq \frac{8}{v_{n}^{3 / 2} \sqrt{n}}\left(\sum_{|k|<n}|\rho(k)|^{3 / 2}\right)^{2}, \\
\kappa_{4}\left(F_{n}\right) & =\mathcal{O}\left(\frac{1}{v_{n}^{2} n}\left(\sum_{|k|<n}|\rho(k)|^{4 / 3}\right)^{3}\right), \\
v_{n} & =-1+2 \sum_{k=0}^{n-1}\left(1-\frac{k}{n}\right) \rho^{2}(k) \asymp \sum_{k=0}^{n-1} \rho^{2}(k) .
\end{aligned}
$$

Proof: We state the proof for the case of positive $\rho$; for negative $\rho$, one only needs to replace $\rho$ by $|\rho|$ in the computations.

Step 1: Computation for the $3^{\text {rd }}$ cumulant.

In Nourdin and Peccati (2015) the following upper bound is proved:

$$
\kappa_{3}\left(F_{n}\right) \leq \frac{8}{v_{n}^{3 / 2} \sqrt{n}}\left(\sum_{|k|<n} \rho(k)^{3 / 2}\right)^{2} .
$$

That reference Nourdin and Peccati (2015) contains the following explicit expression for the third cumulant:

$$
\kappa_{3}\left(F_{n}\right)=\frac{8}{v_{n}^{3 / 2} \sqrt{n}}\left(\frac{1}{n} \sum_{j=0}^{n-1} \sum_{k, l=-j}^{n-1-j} \rho(k) \rho(k-l) \rho(l)\right) .
$$

By discarding the terms with $j>0$, we thus obtain the lower bound:

$$
\kappa_{3}\left(F_{n}\right) \geq \frac{8}{v_{n}^{3 / 2} \sqrt{n}}\left(\sum_{k=0}^{n-1} \sum_{l=0}^{n-1}\left(1-\frac{k}{n}\right) \rho(k) \rho(k-l) \rho(l)\right) .
$$

Since $\rho$ is decreasing near $+\infty, \rho(k+l) \leq \sqrt{\rho(k) \rho(l)}$ for large $k$, $l$. Thus we get

$$
\kappa_{3}\left(F_{n}\right) \geq \frac{8}{v_{n}^{3 / 2} \sqrt{n}} \sum_{k=0}^{n-1}\left(1-\frac{k}{n}\right) \rho(k)^{3 / 2} \sum_{l=0}^{n-1} \rho(l)^{3 / 2}
$$




$$
\geq \frac{8}{v_{n}^{3 / 2} \sqrt{n}} \sum_{l=0}^{n-1} \rho(l)^{3 / 2} \frac{1}{n} \sum_{k=0}^{n-1} S_{k}
$$

where in line (2.4) we used an Abel summation given below in (2.5) (this relation is the subject of Step 2) on the sum $\sum_{k=0}^{n-1}\left(1-\frac{k}{n}\right) \rho(k)^{3 / 2}$, and where $S_{n}=$ $\sum_{l=0}^{n-1} \rho(l)^{3 / 2}$. However, since $\rho$ is positive and decreasing, we have

$$
S_{n}=\mathcal{O}\left(\frac{1}{n} \sum_{k=0}^{n-1} S_{k}\right)
$$

because $S_{2 n} \leq 2 S_{n}$ and $S_{2 n} \geq S_{n}$ for large $n$, so that $\frac{1}{n} \sum_{k=0}^{n-1} S_{n} \geq \frac{1}{2} S_{\left[\frac{n-1}{2}\right]} \geq \frac{1}{4} S_{n}$ for large $n$. That proves the lower bound, and the Theorem's estimate on $\kappa_{3}$, modulo estimate (2.5).

Step 2. Computation of the Abel summation.

To compute

$$
\sum_{k=0}^{n-1}\left(1-\frac{k}{n}\right) \rho(k)^{3 / 2}=S_{n}-\frac{1}{n} \sum_{k=0}^{n-1} k \rho(k)^{3 / 2},
$$

we use Abel's summation-by-parts argument:

$$
\begin{aligned}
\sum_{k=0}^{n-1} k \rho(k)^{3 / 2} & =\sum_{k=1}^{n-1} k\left(S_{k+1}-S_{k}\right)=\sum_{k=1}^{n-1}((k-1)-k) S_{k}+(n-1) S_{n}+\rho(0)^{3 / 2} \\
& =-\sum_{k=1}^{n-1} S_{k}+(n-1) S_{n}+\rho(0)^{3 / 2}
\end{aligned}
$$

so that

$$
\sum_{k=0}^{n-1}\left(1-\frac{k}{n}\right) \rho(k)^{3 / 2}=\frac{1}{n}\left(S_{n}-\rho(0)\right)^{3 / 2}+\sum_{k=1}^{n-1} S_{k} .
$$

Step 3. Upper bound estimation for the $4^{\text {th }}$ cumulant.

In Nourdin and Peccati (2015) the following upper bound is proved: $\kappa_{4}\left(F_{n}\right) \leq$ $\frac{c}{v_{n}^{2} n}\left(\sum_{|k|<n} \rho(k)^{4 / 3}\right)^{3}$. This is our theorem's estimate on $\kappa_{4}\left(F_{n}\right)$.

Step 4. Asymptotic equivalent for the variance.

Let $T(n):=\sum_{k=0}^{n-1} \rho^{2}(k)$. Using a change of summation variables, we find

$$
n v_{n}=\sum_{k=0}^{n-1} \sum_{\ell=0}^{n-1} \rho(k-\ell)^{2}=-n \rho^{2}(0)+2 U(n)
$$

where

$$
U(n):=\sum_{k=0}^{n-1} \rho^{2}(k)(n-k) .
$$

Applying the same arguments as in steps 1 and 2, we find that $U(n) \asymp n T(n)$. With $\rho(0)=1$, this finishes the proof of the theorem. 


\section{A third-moment theorem for normal convergence}

In this section, we establish our third-moment theorem for the normalized centered quadratic variation $F_{n}$ of our stationary Gaussian sequence $\left(X_{n}\right)$, with the assumptions as in Theorem 2.4.

Proposition 3.1. Let $F_{n}$ and $\rho$ be as in Theorem 2.4. Then $n^{1 / 4} \kappa_{4}\left(F_{n}\right)^{3 / 4}=$ $\mathcal{O}\left(\left|\kappa_{3}\left(F_{n}\right)\right|\right)$.

Proof: To lighten the notation, we assume that $\rho$ is positive. The estimates obtained in Theorem 2.4 lead to

$$
\frac{\kappa_{3}\left(F_{n}\right)}{\kappa_{4}\left(F_{n}\right)^{3 / 4}} \geq c n^{1 / 4}\left(\frac{\sum_{|k|<n} \rho(k)^{3 / 2}}{\left(\sum_{|k|<n} \rho(k)^{4 / 3}\right)^{9 / 8}}\right)^{2} .
$$

Hölder's inequality with $p=\frac{9}{4}, q=\frac{9}{5}$ implies

$$
\sum_{|k|<n} \rho(k)^{2 / 3} \rho(k)^{2 / 3} \leq\left(\sum_{|k|<n} \rho(k)^{3 / 2}\right)^{4 / 9}\left(\sum_{|k|<n} \rho(k)^{6 / 5}\right)^{5 / 9}
$$

so that

$$
\left(\frac{\sum_{|k|<n} \rho(k)^{3 / 2}}{\left(\sum_{|k|<n} \rho(k)^{4 / 3}\right)^{9 / 8}}\right)^{2} \geq\left(\frac{\left(\sum_{|k|<n} \rho(k)^{3 / 2}\right)^{2 / 3}}{\left(\sum_{|k|<n} \rho(k)^{6 / 5}\right)^{5 / 6}}\right)^{3 / 2} .
$$

Jensen inequality shows that $\left(\sum_{|k|<n} \rho(k)^{3 / 2}\right)^{2 / 3} \geq\left(\sum_{|k|<n} \rho(k)^{6 / 5}\right)^{5 / 6}$, which allows us to conclude the proof.

This proposition leads to the following.

Theorem 3.2 (Third-moment theorem in total variation and convergence rates). Let $\left(F_{n}\right)_{n \geq 0},\left(v_{n}\right)_{n \geq 0}$, and $\rho$ be as in Theorem 2.4. Denote $N \sim \mathcal{N}(0,1)$. Then the following four statements are equivalent:

(i): $\left(F_{n}\right)_{n \geq 0}$ converges in law towards $\mathcal{N}(0,1)$;

(ii): $\lim _{n \rightarrow \infty} \mathbf{E}\left[F_{n}^{3}\right]=\mathbf{E}\left[N^{3}\right]=0$;

(iii): $\lim _{n \rightarrow \infty} \mathbf{E}\left[F_{n}^{4}\right]=\mathbf{E}\left[N^{4}\right]=3$;

(iv): $\left(\sum_{|k|<n}|\rho(k)|^{3 / 2}\right)^{2}=o\left(v_{n}^{3 / 2} \sqrt{n}\right)$.

Moreover the convergence rate in this situation is $\left|\mathbf{E}\left[F_{n}^{3}\right]\right|$, in the sense that for some $n_{0}>0$

$$
\exists c, C>0: \forall n>n_{0}, c\left|\mathbf{E}\left[F_{n}^{3}\right]\right| \leq d_{T V}\left(F_{n}, N\right) \leq C\left|\mathbf{E}\left[F_{n}^{3}\right]\right| .
$$

We also have the following commensurability for this convergence rate:

$$
\left|\mathbf{E}\left[F_{n}^{3}\right]\right| \asymp \frac{\left(\sum_{|k|<n}|\rho(k)|^{3 / 2}\right)^{2}}{\left(\sum_{|k|<n}|\rho(k)|^{2}\right)^{3 / 2} \sqrt{n}} .
$$


Proof: By the 4th moment theorem (non-quantitative statement in Theorem 1.1), (i) and (iii) are equivalent. By the commensurability relation (2.1) in Theorem 2.4, (ii) and (iv) are equivalent. For the first statement of the theorem, it is now enough to show (ii) and (iii) are equivalent. By Proposition 3.1, we have $\kappa_{4}\left(F_{n}\right)=$ $\mathcal{O}\left(\left|\kappa_{3}\left(F_{n}\right)\right|^{4 / 3} n^{-1 / 3}\right)$, thus if $\kappa_{3}\left(F_{n}\right) \rightarrow 0$ then $\kappa_{4}\left(F_{n}\right) \rightarrow 0$, i.e. (ii) implies (iii). On the other hand, if (iii) holds, by Theorem 1.1, the convergence in (i) holds in total variation, and the theorem's lower bound in (1.1) implies (ii). The second statement of the theorem follows from (1.1) and the estimate $\kappa_{4}\left(F_{n}\right)=\mathcal{O}\left(n^{-1 / 3}\left|\kappa_{3}\left(F_{n}\right)\right|^{4 / 3}\right)$ which shows that $\kappa_{4}\left(F_{n}\right)=o\left(\left|\kappa_{3}\left(F_{n}\right)\right|\right)$. The third statement combines relations (2.1) and (2.3) in Theorem 2.4. The theorem is proved.

The following result can be useful when dealing with non-normal convergence.

Corollary 3.3. If $\rho \notin \ell^{2}(\mathbf{Z})$ then $v_{n} \sim 2 \sum_{k=0}^{n-1}\left(1-\frac{k}{n}\right) \rho^{2}(k)$. This situation holds as soon as $\left(F_{n}\right)_{n \geq 0}$ does not converge in law towards $\mathcal{N}(0,1)$.

Proof: We use the notation and estimates from Step 4 in the proof of Theorem 2.4. Since $U(n) \asymp n T(n)$, we have that $U(n) \asymp n$ iff $T(n)$ converges, i.e. $\rho \in \ell^{2}(\mathbf{Z})$. Therefore if $\rho \notin \ell^{2}(\mathbf{Z})$ (i.e. $\left.T(n) \rightarrow \infty\right)$, then since $U(n) \geq(n / 2) T(n / 2) \gg n$, we can ignore the first term in the equivalent (2.3), proving the asymptotic equivalent of the corollary.

To prove the corollary's second statement, by Theorem 3.2, it is sufficient to show that $\rho \in \ell^{2}(\mathbf{Z})$ implies condition (iv) of the theorem. Since $\rho \in \ell^{2}(\mathbf{Z})$ implies that $v_{n}$ is bounded, we only need to show that $S(n):=\sum_{|k|<n}|\rho(k)|^{3 / 2}=o\left(n^{1 / 4}\right)$. The case of $S(n)$ bounded is trivial, so we assume $S(n)$ is unbounded. For any fixed $\varepsilon>0$, since $\rho \in \ell^{2}$, there exists $n_{1}$ such that $\sum_{k=n_{1}}^{\infty} \rho(k)^{2} \leq \varepsilon$. Also, since $S\left(n_{1}\right)$ is fixed, and $S(n)$ diverges, there exists $n_{2}>n_{1}$ such that for all $n \geq n_{2}$, $S(n) \geq 2 S\left(n_{1}\right)$. Hence we have

$$
S(n)=S(n)-S\left(n_{1}\right)+S\left(n_{1}\right) \leq S(n)-S\left(n_{1}\right)+2^{-1} S(n)
$$

so that

$$
S(n) \leq 2\left(S(n)-S\left(n_{1}\right)\right) .
$$

We have by Jensen's inequality, for all $n \geq n_{2}$,

$$
\begin{aligned}
S(n)-S\left(n_{1}\right) & =2 \sum_{k=n_{1}}^{n-1}|\rho(k)|^{3 / 2}=2\left(n-n_{1}\right)\left[\left(\sum_{k=n_{1}}^{n-1} \frac{1}{n-n_{1}}|\rho(k)|^{3 / 2}\right)^{4 / 3}\right]^{3 / 4} \\
& \leq 2\left(n-n_{1}\right)^{1 / 4}\left[\sum_{k=n_{1}}^{n-1} \frac{1}{n-n_{1}}|\rho(k)|^{2}\right]^{3 / 4} \leq 2 \varepsilon^{3 / 4}\left(n-n_{1}\right)^{1 / 4} .
\end{aligned}
$$

This finishes the proof of the corollary.

\section{Example: sequences with $\log -$ modulated power covariance}

Assume here that the correlation function $\rho$ satisfies

$$
|\rho(n)| \sim n^{2 H-2} \log ^{2 \beta}(n),
$$


for large $n$, with $H \in[0,1]$ and $\beta \in \mathbf{R}$. A stationary sequence $\left(X_{n}\right)_{n \in \mathbf{Z}}$ with this property can easily be constructed as a Gaussian Fourier integral

$$
X_{n}=\int_{S_{1}} \sqrt{q(x)} \cos (x n) W(d x)+\int_{S_{1}} \sqrt{q(x)} \sin (x n) \tilde{W}(d x)
$$

with Fourier coefficients $q(x)=x^{1-2 H} \log ^{-2 \beta}\left(x^{-1}\right)$, where $x$ is the Fourier parameter on the unit circle $S^{1}$, and $W$ and $\tilde{W}$ are independent white noises on $S^{1}$. The details are omitted here; some can be found in the Appendix (see Section 7.1). We also assume, as in the past, that $\rho$ is of constant sign and $|\rho|$ decreases for large $n$. The process constructed above by Gaussian Fourier integral satisfies this assumption. A arbitrary constant scaling factor should be added to the asymptotics of $|\rho|$ (like the $H|2 H-1|$ for $\mathrm{fBm}$ ), but we omit this in this section for notational simplicity.

The case of discrete-time fractional Gaussian noise (fGn), treated in Nourdin and Peccati (2015) and Biermé et al. (2012), falls within the special case of $H \in(0,3 / 4)$ and $\beta=0$. An extension of the fGn is developed in Biermé et al. (2011) contains a correction term added to the the power spectral density of fractional Brownian motion, which decays like a faster power at high frequency. This means that the additional term is treated like a lower-order remainder, for continuous-time data. Strictly speaking, this type of model is not immediately comparable to the discretetime models we consider here. However, a similar study for discrete time could be done, for instance, on correlation structures of the form $|\rho(n)| \sim n^{2 H-2}+r(n)$ where the remainder $r(n)=O\left(n^{2 H-2-\gamma}\right)$ for some $\gamma>0$. Such a class is contained in our assumption (4.1) with $\beta=0$; the corresponding convergence results and speeds of convergence would then depend only on $H$, not $\gamma$. We omit any further discussion of this point for the sake of brevity.

We will see below in Proposition 4.1, that we can cover the case $H=3 / 4$ for any $\beta$, and that within the two presumed "critical thresholds" $H=2 / 3$ and $H=3 / 4$, there arise further "critical log-thresholds" $\beta=-1 / 3$ and $\beta=-1 / 4$ respectively; moreover, the thresholds $H=2 / 3$ and $H=3 / 4$ only give rise to "exotic" convergence rates (i.e. with "log corrections") for certain ranges of the parameter $\beta$. In this sense, the notion of critical value or of phase transition is model-class-dependent. The reader could further convince herself of this by considering a class of processes with $H=2 / 3, \beta=-1 / 3$, and an additional factor $\log \log ^{2 \gamma}(n)$, to find out that the "critical pair" $(H=2 / 3, \beta=-1 / 3)$ which we exhibit harbors further ranges and cutoff values of the parameter $\gamma$, some of which may be considered more exotic than others. In other words, the so-called critical cases do not have any fundamental significance, but just appear as consequences of the models' scaling choices (power-scale in the fractional Brownian example, $\log +$ power-scale in our class of examples).

Also notice that by Proposition 3.1, the asymptotics of $d_{T V}\left(F_{n}, N\right)$ are always given by those of $\kappa_{3}\left(F_{n}\right)$, even when the Breuer-Major theorem fails. This is not a robust result, however. For instance, one can check from the calculations in Biermé et al. (2012) and Nourdin and Peccati (2015) that for $q \geq 3$ and certain values of $H$, the third and fourth cumulants take turns at determining the speed of convergence of $q$ th-power variations of $\mathrm{fBm}$. As soon as the limit of $F_{n}$ is not normal, the question of determining an optimal speed of convergence for $F_{n}$ becomes unresolved. It is known that for $\mathrm{fBm}$ and other self-similar processes, when $H>3 / 4$, the 
normalized quadratic variation converges to a so-called Rosenblatt distribution, which is a law in the second chaos, which depends on the parameter $H$ (see Tudor and Viens (2009) and references therein). Estimating the rate of this convergence from above, for $\mathrm{fBm}$ and for log-modulated processes, is the topic of Sections 5 and 6 ; therein we will see that unlike the case of normal convergence, the rate is determined by the modulation rather than $H$.

Returning to the topic of normal convergence of $F_{n}$, under the asymptotics in (4.1) we now compute the equivalents of the convergence rates exactly, thanks to Theorem 2.4. Let us recall the following well-known result about Bertrand series.

Property 1 (Equivalents of Bertrand series). The series $S_{n}(\alpha, \beta):=\sum_{n>0} n^{\alpha} \log ^{\beta}(n)$ converges if and only if $\alpha<-1$ or $\alpha=-1$ and $\beta<-1$. When the series diverges, we have the following equivalents for its partial sum:

- $S_{n}(-1,-1) \sim \log (\log (n))$;

- $S_{n}(-1, \beta) \sim \frac{1}{\beta+1} \log ^{\beta+1}(n)$ if $\beta>-1$;

- $S_{n}(\alpha, \beta) \sim \frac{1}{\alpha+1} n^{\alpha+1} \log ^{\beta}(n)$ if $\alpha>-1, \beta>-1$.

We may now use this lemma and Theorem 2.4 to obtain the asymptotic order of the third and fourth cumulants, which gives us the rate of convergence to the normal law for $\left(F_{n}\right)$, as a consequence of Theorem 3.2.

Proposition 4.1. With the notation and assumptions as in Theorem 3.2, there are positive constants $c, C$ such that $c M_{n} \leq d_{T V}\left(F_{n}, N\right) \leq C M_{n}$ where

- $M_{n}=\frac{1}{\sqrt{n}}$ if $H<\frac{2}{3}$ or $H=\frac{2}{3}, \beta<-\frac{1}{3}$,

- $M_{n}=\frac{\log (\log (n))^{2}}{\sqrt{n}}$ if $H=\frac{2}{3}, \beta=-\frac{1}{3}$,

- $M_{n}=\frac{1}{\sqrt{n}} \log ^{2(3 \beta+1)}(n)$ if $H=\frac{2}{3}, \beta>-\frac{1}{3}$,

- $M_{n}=n^{6 H-\frac{9}{2}} \log ^{6 \beta}(n)$ if $\frac{2}{3}<H<\frac{3}{4}$ or $H=\frac{3}{4}, \beta<-\frac{1}{4}$,

- $M_{n}=\log ^{-\frac{3}{2}}(n) \log (\log (n))^{-\frac{3}{2}}$ if $H=\frac{3}{4}, \beta=-\frac{1}{4}$,

- $M_{n}=\log ^{-\frac{3}{2}}(n)$ if $H=\frac{3}{4}, \beta>-\frac{1}{4}$.

Since $M_{n} \rightarrow 0$ in all these cases, $d_{T V}\left(F_{n}, N\right) \rightarrow 0$ at the same rates. The normalizing factor $v_{n}$ converges if and only if $\left\{H<\frac{3}{4}\right.$ or $\left.H=\frac{3}{4}, \beta<-\frac{1}{4}\right\}$.

Remark 4.2. If $\left\{H=\frac{3}{4}, \beta \geq-\frac{1}{4}\right\}$ or if $H>3 / 4$, the Breuer-Major theorem fails by definition because $V_{n}:=n^{-1 / 2} \sum_{k=0}^{n-1}\left(X_{k}^{2}-1\right)$ does not converge to a normal (its variance diverges). However the last two cases in the previous proposition show that normal convergence still holds for all cases where $H=3 / 4$. In other words, one must distinguish between (i) a Breuer-Major-type theorem, which attempts to characterize situations where a central limit theorem might hold for partial sums of highly dependent sequences $X_{k}^{2}-1$, where the familiar normalization $n^{-1 / 2}$ can still be used, and (ii) other normal convergences where the dependence of the terms $X_{k}^{2}-1$ is too strong for a central-limit normalization, but hold under a stronger normalization.

Proof of Proposition 4.1: All the computations below are based on the sharp estimates of Theorem 2.4. By Theorem 3.2, it is sufficient to compute a commensurable equivalent of $\kappa_{3}\left(F_{n}\right)$.

Step 1: computing the series $\sum_{|k|<n} \rho(k)^{3 / 2}$. 
Using our class of examples for $\rho$, we have that $\sum_{|k|<n} \rho(k)^{3 / 2}$ diverges if and only if $\sum_{|k|<n}|k|^{3(H-1)} \log ^{3 \beta}(|k|)$ diverges, and is equivalent to this divergent partial sum in that case. Therefore, by the equivalents for Bertrand series, $\sum_{|k|<n} \rho(k)^{3 / 2}$ is equivalent to

$$
\begin{aligned}
& *: \text { a constant } l(H, \beta)>0 \text { if } H<\frac{2}{3} \text { or } H=\frac{2}{3}, \beta<-\frac{1}{3} \\
& *: \log (\log (n)) \text { if } H=\frac{2}{3}, \beta=-\frac{1}{3} \\
& *: \frac{1}{3 \beta+1} \log ^{3 \beta+1} n \text { if } H=\frac{2}{3}, \beta>-\frac{1}{3} \\
& *: \frac{1}{3 H-2} n^{3 H-2} \log ^{3 \beta} n \text { if } H>\frac{2}{3}
\end{aligned}
$$

Step 2: computing $v_{n}$.

Again, by definition of $\rho$, we have that the following quantities are divergent simultaneously and the following equivalences hold in that case: $v_{n} \asymp \sum_{|k|<n} \rho(k)^{2} \sim$ $\sum_{|k|<n}|k|^{4(H-1)} \log ^{4 \beta}(|k|)$. Therefore $v_{n}$ is equivalent to

$$
\begin{aligned}
& *: \text { a constant } l^{\prime}(H, \beta)>0 \text { if } H<\frac{3}{4} \text { or } H=\frac{3}{4}, \beta<-\frac{1}{4} \\
& *: \log (\log (n)) \text { if } H=\frac{3}{4}, \beta=-\frac{1}{4} \\
& *: \frac{1}{3 \beta+1} \log ^{4 \beta+1} n \text { if } H=\frac{3}{4}, \beta>-\frac{1}{4} \\
& *: \frac{1}{4 H-3} n^{4 H-3} \log ^{4 \beta} n \text { if } H>\frac{3}{4}
\end{aligned}
$$

Step 3: computing $\kappa_{3}\left(F_{n}\right)$.

We can now compute a commensurable equivalent for $\kappa_{3}\left(F_{n}\right)$ thanks to relation (2.1).

$$
\begin{aligned}
& *: \quad \kappa_{3}\left(F_{n}\right) \asymp \frac{1}{\sqrt{n}} \text { if } H<\frac{2}{3} \text { or } H=\frac{2}{3}, \beta<-\frac{1}{3} \\
& *: \quad \kappa_{3}\left(F_{n}\right) \asymp \frac{\log (\log (n))^{2}}{\sqrt{n}} \text { if } H=\frac{2}{3}, \beta=-\frac{1}{3} \\
& *: \quad \kappa_{3}\left(F_{n}\right) \asymp \frac{1}{\sqrt{n}} \log ^{2(3 \beta+1)}(n) \text { if } H=\frac{2}{3}, \beta>-\frac{1}{3} \\
& *: \quad \kappa_{3}\left(F_{n}\right) \asymp n^{6 H-\frac{9}{2}} \log ^{6 \beta}(n) \text { if } \frac{2}{3}<H<\frac{3}{4} \text { or } H=\frac{3}{4}, \beta<-\frac{1}{4} \\
& *: \quad \kappa_{3}\left(F_{n}\right) \asymp \log ^{-\frac{3}{2}}(n) \log (\log (n))^{-\frac{3}{2}} \text { if } H=\frac{3}{4}, \beta=-\frac{1}{4} \\
& *: \quad \kappa_{3}\left(F_{n}\right) \asymp \log ^{-\frac{3}{2}}(n) \text { if } H=\frac{3}{4}, \beta>-\frac{1}{4}
\end{aligned}
$$

Theorem 3.2 now allows us to conclude.

\section{Strategy for non-normal convergence}

In Theorem 3.2, we saw that $\left(F_{n}\right)_{n>0}$ converges to a normal if and only if Condition (iv) therein is satisfied. When this condition does not hold, one may wonder what kind of other convergence we could get. The celebrated theorem of Dobrushin and Major (1979); Taqqu (1979) spells out the possible second-chaos limits of $\left(F_{n}\right)_{n \geq 0}$ which occur for slowly-varying perturbations of fGn. In this section, we detail a general methodology, based on the context used by Dobrushin and Major, to determine whether convergence to a second-chaos limit holds.

We are not able to provide general criteria as sharp and as explicit as those which we gave in the previous section for normal convergence; we are not aware of any such works in the literature. However, a classical result of Davydov and Martynova (1989) enables us to define a strategy for establishing speed of convergence in total variation to second-chaos limits. Section 6 shows how to apply this methodology when the slowly-varying terms are logarithmic, similarly to the class of examples presented in Section 4. 
It was recently established in Nourdin and Poly (2012) that the only limits in distribution for sequences in a 2nd Wiener chaos are of the form $\mathcal{L}(N+F)$ where $F$ and $N$ are independent and $F$ is the law of a second-chaos rv, and $N$ is Gaussian. It is conceivable that one could find a choice of law for our sequence $X$ such that $\left(F_{n}\right)_{n \geq 0}$ converges in law to such a convolution. We will avoid such a situation, and investigate how to prove instead that $\left(F_{n}\right)_{n \geq 0}$ converges in law to the law of a second-chaos random variable.

As in Taqqu (1979), we can express our stationary sequence $X$ as a Fourier integral over the unit circle $S^{1} \equiv[-\pi, \pi)$. Let $q$ be the Fourier transform of the even sequence $\rho$ on $\mathbf{Z}$, defined on $S^{1}$ by

$$
q(x):=\sum_{n \in \mathbf{Z}} \rho(n) \cos (n x) .
$$

Assume that $q \in L^{1}\left(S^{1}\right)$. It is known that $q$ is non-negative, with $\int_{S^{1}} q(x) d x=$ $\rho(0)=1$. Since we are interested in sequences $X$ such that $\left(F_{n}\right)_{n \geq 0}$ does not converge to a normal, we will find that typically we have a long memory property, i.e. $\rho \notin \ell^{1}(\mathbf{Z})$, so that the Fourier series defining $q$ is not absolutely convergent. In fact, for $\rho$ decreasing, we saw in Corollary 3.3 that $\rho$ is not even in $\ell^{2}(\mathbf{Z})$. However, if $q \in L^{1}\left(S^{1}\right)$ is given exogenously, the classical theorem on pointwise convergence of Fourier series (see Stein and Shakarchi, 2003, Chapter 3, Theorem 2.1) implies that the Fourier series of $q$ converges to $q$ at all points where $q$ is differentiable. The assumption $q \in L^{1}\left(S^{1}\right)$ and its differentiability will be verified in our examples in Section 6. We will also need to ensure that $\rho$ satisfies a Fourier inversion theorem. Since $\rho \notin \ell^{1}(\mathbf{Z})$, we cannot appeal directly to the classical Fourier inversion theorem, and we must thus check that it holds on a case-by-case basis. In Section 6, we will work with covariances $\rho$ which are defined in terms of their Fourier transform $q$, so that Fourier inversion is automatic.

With $q \in L^{1}\left(S^{1}\right)$ as above, and assuming that Fourier inversion holds for $\rho$, then as for instance in Dobrushin (1979), the following spectral representation holds for the centered Gaussian sequence $X$ whose covariance is $\rho$ : there exists a standard complex-valued white noise $W$ on $S^{1}$ such that

$$
X(k)=\int_{S^{1}} e^{i k x} \sqrt{q(x)} W(d x) .
$$

This appellation for $W$ means that for $x \in[0, \pi], W(d x)=B_{1}(d x)+i B_{2}(d x)$, where $B_{1}$ and $B_{2}$ are two real-valued independent white noise measures on $[0, \pi]$ with scaling constant $(2 \pi)^{-1 / 2}$ (i.e. $\operatorname{Var}\left[B_{i}([0, \pi])\right]=1 / 2$ ), and for every $x \in$ $[0, \pi], W(-d x)=\overline{W(d x)}=B_{1}(d x)-i B_{2}(d x)$. It is helpful to realize that the representation (5.1) is equivalent to

$$
X(k)=\int_{-\pi}^{\pi} \sqrt{q(x)} \cos (k x) W_{1}(d x)+\int_{-\pi}^{\pi} \sqrt{q(x)} \sin (k x) W_{2}(d x)
$$

where $W_{1}$ and $W_{2}$ are i.i.d. real-valued white noises on $[-\pi, \pi]$, standardized so that $\operatorname{Var}\left(W_{i}[-\pi, \pi]\right)=1$. Details on the properties of $W$ are given in the Appendix in Section 7.1. In the remainder of this section and in Section 6, it will be sufficient for us to know the following special case of the isometry formula for double Wiener integrals with respect to $W$ : for $f \in L^{2}\left(\left(S^{1}\right)^{2}, \mathbf{C}\right)$, if $f$ satisfies the Hermitian 
evenness property $f(-x,-y)=\overline{f(x, y)}$, then

$$
\mathbf{E}\left[I_{2}(f)^{2}\right]=\iint_{[-\pi, \pi]^{2}}|f(x, y)|^{2} \frac{d x d y}{(2 \pi)^{2}}=:\|f\|_{L^{2}\left(\left(S^{1}\right)^{2}\right)}^{2} .
$$

See Section 7.1 for a proof. Note in addition that the isometry formula (5.2) for double Wiener integrals against a complex-valued white noise does not contain the usual factor of 2 associated with the isometry property for double Wiener integrals with respect to a real-valued white noise.

From formula (5.1) and the product formula for Wiener integrals, we can write

$$
X(k)^{2}-1=\iint_{\left(S^{1}\right)^{2}} W(d x) W(d y) e^{i k(x+y)} \sqrt{q(x) q(y)} .
$$

Using the stochastic Fubini theorem, justified because $q \in L^{1}\left(S^{1}\right)$ implies that $(k, x, y) \mapsto e^{i k(x+y)} \sqrt{q(x) q(y)}$ is in $L^{2}\left(\left(S^{1}\right)^{2}\right) \times \ell^{2}(\mathbf{Z})$, we get

$$
F_{n}=\frac{1}{\sqrt{n v_{n}}} \iint_{\left(S^{1}\right)^{2}} W(d x) W(d y) \frac{e^{i n(x+y)}-1}{e^{i(x+y)}-1} \sqrt{q(x) q(y)} .
$$

To prove that $\left(F_{n}\right)_{n \geq 0}$ converges in law to a second-chaos distribution, it is sufficient to prove that there exists a Wiener space $(\hat{\Omega}, \hat{\mathcal{F}}, \hat{\mathbf{P}})$ such that for every $n$ there is a random variable $\hat{F}_{n}$ with the same law as $F_{n}$, and another second-chaos random variable $F_{\infty}$ on $(\hat{\Omega}, \hat{\mathcal{F}}, \hat{\mathbf{P}})$ (not dependent on $n$ ), such that $\hat{\mathbf{E}}\left[\left(F_{\infty}-\hat{F}_{n}\right)^{2}\right] \rightarrow 0$. Furthermore, since $d\left(F_{\infty}, F_{n}\right)=d\left(F_{\infty}, \hat{F}_{n}\right)$ for any distance $d$ on the set of laws, to estimate the total-variation distance between the law of $F_{\infty}$ and the law of $F_{n}$, one may rely on a theorem of Davydov and Martynova (1989), by which, if indeed $\left(\hat{F}_{n}\right)_{n>0}$ converges in $L^{2}(\Omega)$ to $F_{\infty}$, then

$$
d_{T V}\left(\hat{F}_{n}, F_{\infty}\right) \leq c_{F_{\infty}}\left(\hat{\mathbf{E}}\left[\left(\hat{F}_{n}-F_{\infty}\right)^{2}\right]\right)^{1 / 4}
$$

where $c_{F_{\infty}}$ is a finite constant depending only on the law of $F_{\infty}$.

We now change variables from $(x, y)$ to $\left(x^{\prime}, y^{\prime}\right):=(x n, y n)$, omitting the primes for parsimony of notation, and we write $I_{n}:=[-\pi n, \pi n)$ for the corresponding scaled circle. The self-similarity of $W(d x)$ with index $1 / 2$ means that $W\left(n^{-1} d x\right)$ has the same law as $n^{-1 / 2} W(d x)$. Therefore the random variable $F_{n}$ has the same law as the variable $\hat{F}_{n}$ defined as follows under a standard complex-valued white noise measure $\hat{W}$ on $\mathbf{R}$ scaled by $(2 \pi)^{-1 / 2}$ (in particular $(\hat{\Omega}, \hat{\mathcal{F}}, \hat{\mathbf{P}})$ is the $\mathbf{C}$-valued Wiener space of $\hat{W}$, and $\left.|\hat{W}(d x)|^{2}=d x /(2 \pi)\right)$ :

$$
\begin{aligned}
\hat{F}_{n}=\frac{1}{\sqrt{n v_{n}}} \iint_{\mathbf{R}^{2}} \mathbf{1}_{I_{n}}(x) \mathbf{1}_{I_{n}}(y) & \sqrt{q\left(\frac{x}{n}\right) q\left(\frac{y}{n}\right)} \\
& \times \frac{1 / n}{e^{i(x+y) / n}-1}\left(e^{i(x+y)}-1\right) \hat{W}(d x) \hat{W}(d y) .
\end{aligned}
$$

We note that $g_{n}: x \mapsto(1 / n)\left(e^{i x}-1\right) /\left(e^{i n x}-1\right)$ converges pointwise and boundedly to the bounded function $g: x \mapsto\left(e^{i x}-1\right) /(i x)$, including at $x=0$; this 
is proved using elementary calculations and the inequality $\left|e^{i x}-1-i x\right| \leq|x|^{2}$ for $|x| \leq 1 / 2$. This implies by dominated (bounded) convergence that $g_{n}$ and $g$ can be interchanged in expressions which are $L^{2}$-convergent. We also recall that we may use the expression $n v_{n}=-n+2 \sum_{k=0}^{n-1}(n-k) \rho^{2}(k)$ which was established in (2.3) in Theorem 2.4.

Now using estimate (5.3) and the isometry property (5.2), the above discussion proves the following criterion for convergence in law of $\hat{F}_{n}$, which includes a possible quantitative estimate. We emphasize that the convergence-in-law portion of this strategy is not new, since it was used by Dobrushin and Major in the case of fGn with slowly varying modulation.

Theorem 1 (Rate of convergence in total variation to the Rosenblatt law). Let $\left(F_{n}\right)_{n \geq 0}$ be the sequence of normalized quadratic variations of a centered stationary Gaussian sequence $\left(X_{n}\right)$ with covariance $\rho$, i.e. $F_{n}:=V_{n} / \sqrt{v_{n}}$, where $V_{n}:=n^{-1 / 2} \sum_{k=0}^{n-1}\left(X_{k}^{2}-1\right)$, and $v_{n}:=E\left[V_{n}^{2}\right]=n^{-1} \sum_{k=0}^{n-1} \sum_{\ell=0}^{n-1} \rho(k-\ell)^{2}=$ $-1+2 \sum_{k=0}^{n-1}(1-k / n) \rho^{2}(k)$. Assume that the spectral density $q$ of $X$, defined by

$$
q(x):=\sum_{n \in \mathbf{Z}} \rho(n) \cos (n x)
$$

exists as a member of $L^{1}([-\pi, \pi))$ Assume that Fourier inversion holds, i.e. that $\int_{-\pi}^{\pi} e^{i k x} q(x) \frac{d x}{2 \pi}=\rho(k)$ for all $k \in \mathbf{Z}$. Assume $\rho(0)=1$. Let

$$
I_{n}:=[-\pi n, \pi n), g(x):=\frac{e^{i x}-1}{i x} .
$$

Assume there exists a real function $f \in L_{l o c}^{2}\left(\mathbf{R}^{2}\right)$ which is even in both variables and such that $(x, y) \mapsto\left(n v_{n}\right)^{-1 / 2} \sqrt{q\left(\frac{x}{n}\right) q\left(\frac{y}{n}\right)} \mathbf{1}_{I_{n}}(x) \mathbf{1}_{I_{n}}(y) g(x+y)$ converges in $L^{2}\left(\mathbf{R}^{2}\right)$ to $f(x, y) g(x+y)$.

Then $F_{n}$ converges in law to the law of a second-chaos variable $F_{\infty}$, and we have the representation

$$
F_{\infty}=\iint_{\mathbf{R}^{2}} f(x, y) \frac{e^{i(x+y)}-1}{i(x+y)} W(d x) W(d y)
$$

where $W$ is a complex white noise on $\mathbf{R}$ with scale determined by $|W(d x)|^{2}=$ $d x /(2 \pi)$.

Moreover, with $c_{F_{\infty}}$ the constant in (5.3), the speed of convergence in total variation is bounded above as

$$
\begin{aligned}
d_{T V}\left(F_{n}, F_{\infty}\right)^{4} & \leq\left(c_{F_{\infty}}\right)^{4} \iint_{\mathbf{R}^{2}} \mid \mathbf{1}_{I_{n}}(x) \mathbf{1}_{I_{n}}(y) \frac{1}{\sqrt{n v_{n}}} \sqrt{q\left(\frac{x}{n}\right) q\left(\frac{y}{n}\right)} \\
& \times \frac{n^{-1}\left(e^{i(x+y)}-1\right)}{e^{i(x+y) / n}-1}-\left.f(x, y) \frac{e^{i(x+y)}-1}{i(x+y)}\right|^{2} d x d y
\end{aligned}
$$

Proof: See above development.

Remark 1. The examples in Section 6 satisfy the assumption on $q$ and $\rho$ in Theorem 1.

Remark 2. The last inequality above, and Corollary 1 below, hold with $y$ replaced by $-y$ since $f$ is even in each variable, and $q$ and $I_{n}$ are even. 
Remark 3. Under the assumptions of Theorem 1, the law of $\left(F_{n}\right)_{n>0}$ cannot converge to a normal law. Consequently by Theorem 3.2, the other three equivalent conditions (ii), (iii), (iv) therein fail. In particular, by Corollary 3.3, $\rho \notin \ell^{2}(\mathbf{Z})$.

The following corollary is useful to estimate the speed of convergence in Theorem 1. It enables one to introduce a trade-off between the speed of convergence of the improper integral defining $\|f g\|_{L^{2}\left(\mathbf{R}^{2}\right)}^{2}$ and the speed of convergence of $\sqrt{q\left(\frac{x}{n}\right) q\left(\frac{y}{n}\right) n^{-1} / v_{n}}$ to $f$.

Corollary 1. Under the assumptions and notation of Theorem 1 , for any $\alpha \in(0,1)$, with $h(x):=\min \left(1,|x|^{-1}\right)$, and $n v_{n}=-n+2 \sum_{k=0}^{n-1}(n-k) \rho^{2}(k)$,

$$
\begin{aligned}
\frac{1}{\left(c_{F_{\infty}}\right)^{4}} d_{T V}\left(F_{n}, F_{\infty}\right)^{4} & \leq 5 \iint_{\mathbf{R}^{2} \backslash\left(I_{n} \alpha\right)^{2}}|f(x, y)|^{2} h^{2}(x+y) d x d y \\
& +16 \iint_{\left(I_{n}\right)^{2}}\left|\sqrt{\frac{q\left(\frac{x}{n}\right) q\left(\frac{y}{n}\right)}{n v_{n}}}-f(x, y)\right|^{2} h^{2}(x+y) d x d y \\
& +\frac{4}{n^{2-2 \alpha}} \iint_{\mathbf{R}^{2}}|f(x, y)|^{2} h^{2}(x+y) d x d y
\end{aligned}
$$

Proof: Write $g(x)=\left(e^{i x}-1\right) /(i x)$ as above, and

$$
f_{n}(x, y)=\left(n v_{n}\right)^{-1 / 2} \sqrt{q\left(\frac{x}{n}\right) q\left(\frac{y}{n}\right)} ; \quad g_{n}(x)=\frac{n^{-1}\left(e^{i x}-1\right)}{e^{i x / n}-1} .
$$

The function $g$ is bounded by $2 h(x)$. Elementary calculations with $n \geq 3$ and $x, y \in I_{n}$ lead to

$$
\left|g_{n}(x+y)-g(x+y)\right| \leq \frac{|g(x+y)|}{1+n /|x+y|} \leq \frac{2 h(x+y)}{1+n /|x+y|} .
$$

From Theorem 1,

$$
\begin{aligned}
& d_{T V}\left(F_{n}, F_{\infty}\right) \\
& \leq \iint_{\mathbf{R}^{2} \backslash\left(I_{n}\right)^{2}}|f(x, y)|^{2} h^{2}(x+y) d x d y \\
& +\iint_{\left(I_{n}\right)^{2}}\left(\left|f_{n}(x, y)-f(x, y)\right|^{2}\left|g_{n}(x+y)\right|^{2}+|f(x, y)|^{2}\left|g_{n}(x+y)-g(x+y)\right|^{2}\right) d x d y \\
& \leq \iint_{\mathbf{R}^{2} \backslash\left(I_{n}\right)^{2}}|f(x, y)|^{2} h^{2}(x+y) d x d y \\
& +16 \iint_{\left(I_{n}\right)^{2}}\left|f_{n}(x, y)-f(x, y)\right|^{2} h^{2}(x+y) d x d y \\
& +\iint_{\left(I_{n}\right)^{2}}\left|g_{n}(x+y)-g(x+y)\right|^{2}|f(x, y)|^{2} d x d y .
\end{aligned}
$$

All three terms above converge to 0 , the first two by assumption, the last by dominated convergence. However, to derive quantitative estimates, it is best to exploit 
relation (5.7) more specifically. Therefore, for $\alpha \in(0,1)$ fixed, we write

$$
\begin{aligned}
& \iint_{\left(I_{n}\right)^{2}}\left|g_{n}(x, y)-g(x, y)\right|^{2}|f(x, y)|^{2} d x d y \\
& \left.=\iiint_{\left(I_{n}\right)^{2} \backslash\left(I_{n} \alpha\right)^{2}}+\iint_{\left(I_{n}\right)^{2}}\right)\left|g_{n}(x, y)-g(x, y)\right|^{2}|f(x, y)|^{2} d x d y \\
& \leq 4 \iint_{\mathbf{R}^{2} \backslash\left(I_{n} \alpha\right)^{2}}|f(x, y)|^{2} h^{2}(x+y) d x d y+\frac{4}{n^{2-2 \alpha}} \iint_{\mathbf{R}^{2}}|f(x, y)|^{2} h^{2}(x+y) d x d y .
\end{aligned}
$$

The corollary easily follows.

\section{Example: log-modulation and second-chaos limits}

We have in mind the same class of examples as in Section 4. If $H>\frac{3}{4}$, one gets, by relation (2.1) in Theorem 2.4 , that $\kappa_{3}\left(F_{n}\right)$ converges to a non-zero constant. Theorem 3.2 then proves that $F_{n}$ cannot converge to a normal law. We now study the possible convergence of $F_{n}$ to non-normal laws.

In order to streamline the presentation, since our strategy is to use Corollary 1 , it turns out to be more convenient to make assumptions on $q$ and derive corresponding estimates on $\rho$ and other quantities of interest. The reader may see below, by comparing our definition of $q$ in (6.1) and the estimate on $\rho$ in Proposition 1, that assumptions on $q$ and $\rho$ of log-modulated power type are asymptotically equivalent.

Let $\beta \geq 0$ and $H \in(3 / 4,1)$. Consider the positive function $q$ on the unit circle $S^{1}=[-\pi, \pi]$ defined, except at $x=0$, by

$$
q(x):=C_{H, \beta}|x|^{1-2 H} \log ^{2 \beta}\left(\frac{e \pi}{|x|}\right) .
$$

The constant $C_{H, \beta}$ is chosen in such a way that $\int_{-\pi}^{\pi} q(x) d x /(2 \pi)=1$, i.e.

$$
C_{H, \beta}:=2 \pi / \int_{-\pi}^{\pi}|x|^{1-2 H} \log ^{2 \beta}\left(\frac{e \pi}{|x|}\right) d x,
$$

in order to stay with the assumption that our stationary sequence has unit variance, but other normalizing constants pose no additional difficulty. The case $\beta=0$ corresponds to fGn. The case $\beta<0$ has slightly different properties than the case $\beta \geq 0$, and requires further computations; we omit it for the sake of conciseness. To simplify the notation, we introduce

$$
L(y):=\log ^{2 \beta}(|y|)
$$

and notice that $L(e \pi /|x|) \geq 1$ for all $x \in[-\pi, \pi]$. Moreover, $q$ is in $L^{1}\left(S^{1}, d x\right)$ and is $C^{\infty}$ everywhere except at 0 , and therefore $q$ coincides with the Fourier series of its Fourier inverse $\rho$; in other words, the stationary Gaussian process $X$ with covariance function $\rho$ given by

$$
\rho(k):=\frac{1}{2 \pi} \int_{-\pi}^{\pi} q(x) \cos (k x) d x
$$

has spectral density $q$. The relation (6.3), which is our definition of $\rho$, serves as the Fourier inversion property required for applying Theorem 1 and its corollary. The 
other needed assumption is $q \in L^{1}\left(S^{1}\right)$, which holds since $H<1$. We recompute $\rho$ by changing variables and using the definition of $q$, to get

$$
\rho(k)=\frac{C_{H, \beta}}{2 \pi} k^{2 H-2} \int_{-k \pi}^{k \pi}|x|^{1-2 H} \cos (x) L\left(k \frac{e \pi}{|x|}\right) d x .
$$

Here normalizations are such that $\rho(0)=1$. The asymptotics of $\rho$ are the following.

Proposition 1. With $\rho$ in (6.4), with $C_{H, \beta}$ in (6.2) and with

$$
K_{H}:=\frac{1}{\pi} \int_{0}^{\infty}|x|^{1-2 H} \cos (x) d x=2 \Gamma(2-2 H) \cos (\pi(1-H)),
$$

we have for large $k$

$$
\rho(k)=C_{H, \beta} K_{H} L(k) k^{2 H-2}\left(1+\mathcal{O}\left(\frac{1}{L(k)}\right)\right) .
$$

The proof of this proposition is given in Section 7.2 in the Appendix. The next proposition, which gives the behavior of $n v_{n}=\operatorname{Var}\left(\sum_{k=0}^{n-1} X_{k}^{2}\right)$ using Proposition 1, is also proved in the Appendix, in Section 7.3. Recall from (2.3) in Theorem 2.4 that $n v_{n}=n+2 \sum_{k=1}^{n-1}(n-k) \rho^{2}(k)$.

Proposition 2. With $\rho$ in (6.4) and $n v_{n}=n+2 \sum_{k=1}^{n-1}(n-k) \rho^{2}(k)$, for $n$ large

$$
n v_{n}=\left(C_{H, \beta}\right)^{2} K_{H}^{\prime} n^{4 H-2} L^{2}(n)\left(1+\mathcal{O}\left(\frac{1}{\log n}\right)\right)
$$

where $C_{H, \beta}$ is given in (6.2) and

$$
K_{H}^{\prime}:=\frac{(2 \Gamma(2-2 H) \cos (\pi(1-H)))^{2}}{(4 H-2)(4 H-3)} .
$$

With Proposition 2 in hand, we can use Corollary 1 to establish a speed of convergence result for the Dobrushin-Major theorem.

Theorem 2. Let $\left(F_{n}\right)_{n \geq 0}$ be the sequence of normalized quadratic variations of a centered stationary Gaussian sequence $\left(X_{n}\right)$ with unit variance, i.e. $F_{n}:=V_{n} / \sqrt{v_{n}}$, where $V_{n}:=n^{-1 / 2} \sum_{k=0}^{n-1}\left(X_{k}^{2}-1\right)$, and $v_{n}:=E\left[V_{n}^{2}\right]=n^{-1} \sum_{k=0}^{n-1} \sum_{\ell=0}^{n-1} \rho(k-\ell)^{2}$, with the covariance $\rho$ of $X$ given by (6.4). Assume $H \in(3 / 4,1)$ and $\beta \geq 0$. Then $F_{n}$ converges in law to the law of a second-chaos variable $F_{\infty}$, and we have the representation

$$
F_{\infty}=\iint_{\mathbf{R}^{2}} \frac{|x y|^{H-1 / 2}}{\sqrt{K_{H}^{\prime}}} \frac{e^{i(x+y)}-1}{i(x+y)} W(d x) W(d y)
$$

where $W$ is a complex-valued white noise on $\mathbf{R}$ with scale determined by $|W(d x)|^{2}=$ $d x /(2 \pi)$ and $K_{H}^{\prime}$ is given in Proposition 2. Moreover, the speed of convergence in total variation is bounded above as

$$
d_{T V}\left(F_{n}, F_{\infty}\right) \leq \frac{c}{\log ^{1 / 2} n}
$$

where the positive constant $c$ depends only on $H$ and $\beta$.

Before concluding our article with the proof of this theorem, we rephrase the result to match the usual normalizations found in the literature when $H>3 / 4$. 
Remark 4. According to Theorem 2, with $\left(X_{n}\right)$ the correlated sequence of standard normal random variables defined therein, the sequence

$$
\frac{\sum_{k=0}^{n-1}\left(X_{k}^{2}-1\right)}{n^{2 H-1}}
$$

converges in distribution to the law of the second-chaos variable represented by

$$
C_{H, \beta} \iint_{\mathbf{R}^{2}}|x y|^{H-1 / 2} \frac{e^{i(x+y)}-1}{i(x+y)} W(d x) W(d y)
$$

where $W$ is a complex-valued white noise on $\mathbf{R}$ with scale determined by $|W(d x)|^{2}=$ $d x /(2 \pi)$ and $C_{H, \beta}$ is given in (6.2). The speed of convergence in total variation is of order $\log ^{-1 / 2} n$ for any $\beta>0$ and any $H \in(3 / 4,1)$. When $\beta=0$, this speed can be improved to $n^{3 / 4-H}$ as can be seen in Nourdin and Peccati (2012, Theorem 7.4.5).

Proof of Theorem 2. Since, as mentioned above, $q$ and $\rho$ satisfy the assumptions of Theorem 1, according to Corollary 1, and using its notation, it is sufficient to prove that, with $f(x, y)=|x y|^{H-1 / 2} / \sqrt{K_{H}^{\prime}}$, the three terms in lines (5.4), (5.5), and (5.6) all converge to 0 at least as fast as $\log ^{-2} n$. In all calculations below, it will be convenient to change variables and replace $y$ by $-y$; this only entails replacing $h(x+y)$ by $h(x-y)$; all other expressions are invariant by this change of variables since $I_{n}$ and $q$ are even and $f$ is even in $y$; see Remark 2.

Step 1. We use Proposition 2 to handle the term in line (5.5). With $\gamma=2-2 H$, this term is bounded above by a constant times

$$
\begin{aligned}
\mathcal{I}(2) & : \quad=\iint_{\left(I_{n}\right)^{2}}\left|\sqrt{\frac{q\left(\frac{x}{n}\right) q\left(\frac{y}{n}\right)}{n v_{n}}}-f(x, y)\right|^{2} h^{2}(x-y) d x d y \\
& =\iint_{\left(I_{n}\right)^{2}} \frac{|x y|^{\gamma-1}}{K_{H}^{\prime}}\left|\frac{\sqrt{L\left(\frac{e \pi n}{x}\right) L\left(\frac{e \pi n}{y}\right)}}{\left(1+\mathcal{O}\left(\frac{1}{\log n}\right)\right) L(n)}-1\right|^{2} \min \left(1,|x-y|^{-2}\right) d x d y .
\end{aligned}
$$

We can write

$$
\frac{L\left(\frac{e \pi n}{x}\right)}{L(n)}=\left(1-\frac{\log |x|}{\log (e \pi n)}\right)^{2 \beta} .
$$

In order to use a uniform bound on the first order Taylor expansion for this expression, we will fix $\delta \in(0,1)$ and consider $u \in[-\delta, 1]$; then there is a positive finite constant $c=c(\beta, \delta)$ such that for large $n,\left|(1-u)^{\beta}-1+\beta u\right| \leq c u^{2}$. Hence for $|x| \in\left[n^{-\delta}, \pi n\right]$ and $n$ large enough,

$$
\frac{\sqrt{L\left(\frac{e \pi n}{x}\right) L\left(\frac{e \pi n}{y}\right)}}{\left(1+\mathcal{O}\left(\frac{1}{\log n}\right)\right) L(n)}=1+\mathcal{O}\left(\frac{1}{\log n}\right)-\beta \frac{\log |x|+\log |y|}{\log n}+c \frac{\log ^{2}|x|+\log ^{2}|y|}{\log ^{2} n} .
$$

Step 1.1. For $n$ large enough, by symmetry, the portion of $\mathcal{I}(2)$ for $|x| \geq n^{-\delta}$ and $|y| \geq n^{-\delta}$ is bounded above by a constant times

$$
\int_{0}^{\pi n} \int_{0}^{x}(x y)^{\gamma-1}\left(\frac{1}{\log n}+\frac{|\log x|+|\log y|}{\log n}+\frac{\log ^{2} x+\log ^{2} y}{\log ^{2} n}\right)^{2} \min \left(1,|x-y|^{-2}\right) d y d x .
$$


Since $(x y)^{\gamma-1}\left(1+|\log x|+\log ^{2} x\right)\left(1+|\log y|+\log ^{2} y\right) \mathbf{1}_{0 \leq y \leq x}$ is integrable for $x$ near 0 , the contribution of the above integral for $x \in[0,2]$ is $\mathcal{O}\left(\log ^{-2} n\right)$. Therefore, by separating this set and the diagonal set $x-1<y<x$ from the rest, the above integral is bounded above by a constant times

$$
\begin{aligned}
& \frac{1}{\log ^{2} n}+\int_{2}^{\pi n} \int_{x-1}^{x} y^{\gamma-1} \frac{\log ^{2} x+\log ^{2} y+\log ^{4} x+\log ^{4} y}{\log ^{2} n} d y d x \\
& +\int_{2}^{\pi n} \int_{0}^{x-1} y^{\gamma-1}(x-y)^{-2} \frac{\log ^{2} x+\log ^{2} y+\log ^{4} x+\log ^{4} y}{\log ^{2} n} d y d x
\end{aligned}
$$

This is itself bounded above by

$$
\begin{aligned}
& \frac{1}{\log ^{2} n}+\frac{4}{\log ^{2} n} \int_{2}^{\pi n}(x-1)^{\gamma-1} \log ^{4} x d x \\
& +\frac{1}{\log ^{2} n} \int_{2}^{\pi n} \int_{0}^{1} y^{\gamma-1}(x-y)^{-2}\left(\log ^{2}(x / y)+\log ^{4}(x / y)\right) d y d x \\
& +\frac{4}{\log ^{2} n} \int_{2}^{\pi n} \log ^{4} x \int_{1}^{x-1} y^{\gamma-1}(x-y)^{-2} d y d x \\
& \leq \frac{1}{\log ^{2} n}+\frac{4}{\log ^{2} n}\left(\int_{1}^{\infty} x^{\gamma-1} \log ^{4}(x+1) d x\right) \\
& +\frac{1}{\log ^{2} n} \int_{2}^{\pi n} x^{\gamma-2} \int_{0}^{1 / x} z^{\gamma-1}(1-z)^{-2}\left(\log ^{2} z+\log ^{4} z\right) d z d x \\
& +\frac{4}{\log ^{2} n} \int_{2}^{\pi n} x^{\gamma-2} \log ^{4} x \int_{1 / x}^{1-1 / x} z^{\gamma-1}(1-z)^{-2} d y d x \\
& \leq \frac{c}{\log ^{2} n}\left(\int_{2}^{\pi n} x^{\gamma-2} x^{-\gamma+\varepsilon} d x+\int_{2}^{\pi n} x^{\gamma-2} \log ^{4} x d x\left[4 \int_{1 / x}^{1 / 2} z^{\gamma-1} d z+4 \int_{1 / x}^{1 / 2} z^{-2} d z\right]\right) \\
& \leq \frac{c}{\log ^{2} n}\left(1+\int_{2}^{\pi n} x^{\gamma-2}\left(x^{-\gamma+\varepsilon}+x^{-\gamma}+x^{-1}\right) \log ^{4} x d x\right)
\end{aligned}
$$

for any constant $\varepsilon>0$, where $c$ depends only on $\gamma$ and $\varepsilon$. By choosing $\varepsilon<\gamma$, we get that this portion of $\mathcal{I}(2)$ goes to 0 as fast as $\log ^{-2} n$.

Step 1.2. For $n$ large enough, by symmetry, the portion of $\mathcal{I}(2)$ for $|x| \leq n^{-\delta}$ or $|y| \leq n^{-\delta}$ is bounded above by a constant times

$$
\begin{aligned}
& \int_{0}^{\pi n} y^{\gamma-1} d y \int_{0}^{n^{-\delta}} x^{\gamma-1}\left(1+\frac{L\left(\frac{e \pi n}{x}\right)}{L(n)}+\frac{L\left(\frac{e \pi n}{y}\right)}{L(n)}\right) \min \left(1,|x-y|^{-2}\right) d x \\
& \leq \int_{0}^{n^{-\delta}} y^{\gamma-1} d y \int_{0}^{n^{-\delta}} x^{\gamma-1}\left(2+L\left(\frac{1}{x}\right)+L\left(\frac{1}{y}\right)\right) d x \\
& +\int_{n^{-\delta}}^{1} y^{\gamma-1} d y \int_{0}^{n^{-\delta}} x^{\gamma-1}\left(3+L\left(\frac{1}{x}\right)\right) d x \\
& +\int_{n^{-\delta}}^{\pi n} y^{\gamma-1} \frac{4}{y^{2}} d y \int_{0}^{n^{-\delta}} x^{\gamma-1}\left(3+L\left(\frac{1}{x}\right)\right) d x .
\end{aligned}
$$


Therefore, for any $\varepsilon>0$, and $n$ large enough, the above expression is bounded above by a constant times

$$
\begin{aligned}
& \int_{0}^{n^{-\delta}} y^{\gamma-1} d y n^{-\gamma \delta}\left(2+L\left(\frac{1}{y}\right)+n^{\varepsilon}\right)+\int_{n^{-\delta}}^{1} y^{\gamma-1} d y n^{-\gamma \delta}\left(3+n^{\varepsilon}\right) \\
& +\int_{1}^{\pi n} y^{\gamma-3} d y n^{-\gamma \delta}\left(3+n^{\varepsilon}\right) \\
& \leq \gamma^{-1} n^{-\gamma \delta}\left(2+n^{\varepsilon}+n^{2 \varepsilon}\right)+4 \gamma^{-1} n^{-\gamma \delta+\varepsilon}+4(2-\gamma)^{-1} n^{-\gamma \delta+\varepsilon} \\
& \leq c n^{-\gamma \delta+2 \varepsilon}
\end{aligned}
$$

for some constant $c$ depending only on $\gamma$ and $\varepsilon$. For $\varepsilon<\gamma \delta / 2$, we get that this portion of $\mathcal{I}(2)$ goes to 0 faster than $\log ^{-2} n$. Hence $\mathcal{I}(2)=\mathcal{O}\left(\log ^{-2} n\right)$.

Step 2. We now consider the term in line (5.4). By symmetry, this is bounded above by a constant times

$$
\mathcal{I}(1):=\int_{0}^{\infty} x^{\gamma-1} \int_{n^{\alpha}}^{\infty} y^{\gamma-1} \min \left(1,|x-y|^{-2}\right) d y d x .
$$

That is in turn bounded above by

$$
\int_{0}^{n^{\alpha}-1} x^{\gamma-1}\left(\int_{n^{\alpha}}^{\infty} y^{\gamma-1}(y-x)^{-2} d y\right) d x+2 \int_{n^{\alpha}-1}^{\infty} x^{\gamma-1} \int_{n^{\alpha}-1}^{x} y^{\gamma-1} \min \left(1,|x-y|^{-2}\right) d y d x
$$

which is itself bounded above by

$$
\begin{aligned}
& n^{\alpha(\gamma-1)} \int_{0}^{n^{\alpha}-1} x^{\gamma-1}\left(n^{\alpha}-x\right)^{-1} d x+2 \int_{n^{\alpha}-1}^{\infty} x^{\gamma-1} \int_{n^{\alpha}-1}^{x-1} y^{\gamma-1}(x-y)^{-2} d y d x \\
& +2 \int_{n^{\alpha}-1}^{\infty} x^{\gamma-1} \int_{x-1}^{x} y^{\gamma-1} d y d x \\
& \leq n^{\alpha(\gamma-1)}\left(\frac{2}{n^{\alpha}} \int_{0}^{n^{\alpha} / 2} x^{\gamma-1} d x+\left(\frac{2}{n^{\alpha}}\right)^{\gamma-1} \int_{1}^{n^{\alpha} / 2} x^{-1} d x\right) \\
& +2 \int_{n^{\alpha}-1}^{\infty} y^{\gamma-1} \int_{x+1}^{\infty}(x-y)^{-2} d x d y+2 \int_{n^{\alpha}-1}^{\infty}(x-1)^{2 \gamma-2} d x \\
& =n^{2 \alpha(\gamma-1)}\left(\frac{2^{1-\gamma}}{\gamma}+2^{\gamma-1} \log \left(\frac{n^{\alpha}}{2}\right)\right)+\frac{2}{1-\gamma}\left(n^{\alpha}-1\right)^{\gamma-1}+\frac{2}{1-2 \gamma}\left(n^{\alpha}-2\right)^{2 \gamma-1} .
\end{aligned}
$$

Since $\gamma=2-2 H$ and $H \in(3 / 4,1)$, we have $0>2 \gamma-1>\gamma-1>2 \gamma-2$, and thus the last expression above is a $\mathcal{O}\left(n^{-\alpha(1-2 \gamma)}\right)$ which goes to 0 faster than $\log ^{-2} n$. I.e. $\mathcal{I}(1)=\mathcal{O}\left(n^{-\alpha(1-2 \gamma)}\right) \ll \log ^{-2} n$.

Step 3. Finally we consider the term in line (5.6). By symmetry, this is bounded above by a constant times

$$
\mathcal{I}(3):=\frac{1}{n^{2-2 \alpha}} \int_{0}^{\infty} x^{\gamma-1} \int_{0}^{x} y^{\gamma-1} \min \left(1,(x-y)^{-2}\right) d y d x
$$

To show that this $\mathcal{I}(3)=o\left(\log ^{-2} n\right)$ it is sufficient to prove that the integral above is finite. Since $\gamma-1>-1$, The portion of that integral corresponding to $x \in[0,2]$ 
is finite. We thus only need to study the portion corresponding to $x>2$ :

$$
\begin{aligned}
& \int_{2}^{\infty} x^{\gamma-1} \int_{0}^{x} y^{\gamma-1} \min \left(1,(x-y)^{-2}\right) d y d x \\
& \leq \int_{2}^{\infty} x^{\gamma-1}\left(\left(\frac{x}{2}\right)^{-2} \int_{0}^{x / 2} y^{\gamma-1} d y+\left(\frac{x}{2}\right)^{\gamma-1} \int_{x / 2}^{x-1}(x-y)^{-2} d y+\int_{x-1}^{x} y^{\gamma-1} d y\right) d x \\
& \leq \int_{2}^{\infty} x^{\gamma-1}\left(\left(\frac{x}{2}\right)^{-2} \gamma^{-1}\left(\frac{x}{2}\right)^{\gamma}+\left(\frac{x}{2}\right)^{\gamma-1}\left(\int_{1}^{\infty} y^{-2} d y\right)+(x-1)^{\gamma-1}\right) d x \\
& \leq \gamma^{-1} 2^{2-\gamma} \int_{2}^{\infty} x^{2 \gamma-3} d x+2^{2-\gamma} \int_{1}^{\infty} x^{2 \gamma-2} d x
\end{aligned}
$$

This is finite, given that $H>3 / 4 \Longrightarrow 2 \gamma-2<-1$. The proof of the theorem is complete.

\section{Appendix: technical elements used in Sections 5 and 6.}

7.1. Representations of stationary Gaussian processes using the complex-valued white noise measure. For the reader's convenience, and for the sake of being selfcontained to some extent, we briefly recall the construction and properties of the complex-valued white noise $W$. As indicated in Section $5, W$ is the independently scattered $\mathbf{C}$-valued centered Gaussian measure on $S^{1}=[-\pi, \pi]$ such that for $x \in[0, \pi], W(d x)=B_{1}(d x)+i B_{2}(d x)$, where $B_{1}$ and $B_{2}$ are two real-valued independent white noise measures on $[0, \pi]$ such that $\operatorname{Var}\left[B_{i}([0, \pi])\right]=1 / 2$, and for every $x \in[0, \pi], W(-d x)=\overline{W(d x)}=B_{1}(d x)-i B_{2}(d x)$.

This definition of $W$, where one notes that unlike in the real case, the restrictions of $W$ to $[-\pi, 0]$ and to $[0, \pi]$ are not independent, implies the following properties, using the usual shorthand differential notation for Itô's rule: for all $x \in[0, \pi]$,

- first Itô rule: $W(d x) W(-d x)=W(d x) \overline{W(d x)}=|W(d x)|^{2}=d x /(2 \pi)$,

- second Itô rule: $W(d x) W(d x)=W(d x)^{2}=0$,

- $W(d x)$ and $W(d y)$ are independent for $x \neq y$ and $x y \geq 0$,

For $X$ defined as in (5.1) using a non-negative function $q \in L^{1}\left(S^{1}\right)$, i.e. for $k \in \mathbf{Z}$,

$$
X(k)=\int_{S^{1}} e^{i k x} \sqrt{q(x)} W(d x)
$$

we can rewrite this expression by expanding $W$ according to its definition above: for all $k \in \mathbf{Z}$,

$$
\begin{aligned}
X(k) & =2 \int_{0}^{\pi} \sqrt{q(x)} \cos (k x) B_{1}(d x)-2 \int_{0}^{\pi} \sqrt{q(x)} \sin (k x) B_{2}(d x) \\
& =\int_{-\pi}^{\pi} \sqrt{q(x)} \cos (k x) W_{1}(d x)+\int_{-\pi}^{\pi} \sqrt{q(x)} \sin (k x) W_{2}(d x)
\end{aligned}
$$

where the second equality is in law, with $W_{1}$ and $W_{2}$ two independent real-valued white noise measures on $[-\pi, \pi]$ with scale determined by $\operatorname{Var}\left[W_{i}([-\pi, \pi])\right]=1$.

Representation (7.2) is more commonly found in the literature than Representation (7.1). From either representation, it is evident that $X$ is real valued. From (5.1), one can check that $X$ has the announced covariance, as follows. Using the fact that $X$ is real-valued, and the product rule for Wiener integrals, we have $X(k) X(l)=X(k) \overline{X(l)}$, which is the sum of a mean-zero second-chaos variable 
and of a constant formally expressed as $\int_{S^{1}} e^{i k x} \sqrt{q(x)} W(d x) e^{-i l x} \sqrt{q(x)} \overline{W(d x)}$. Thus, by Itô's rule,

$$
\mathbf{E}[X(k) X(l)]=\int_{-\pi}^{\pi} e^{i(k-l) x} q(x) \frac{d x}{2 \pi}=\rho(k-l),
$$

where the last equality assumes that Fourier inversion holds for $\rho$. We can extend this type of calculation in general to express the isometry property in the second chaos of the complex-valued $W$. For a function $f \in L^{2}\left([0, \pi]^{2}, \mathbf{C}\right)$ we can write

$$
\begin{aligned}
I_{2}(f)= & \iint_{[0, \pi]^{2}} f(x, y) W(d x) W(d y)+\iint_{[0, \pi] \times[-\pi, 0]} f(x, y) W(d x) W(d y) \\
& +\iint_{[-\pi, 0]^{2}} f(x, y) W(d x) W(d y)+\iint_{[-\pi, 0] \times[0, \pi]} f(x, y) W(d x) W(d y) \\
= & \iint_{[0, \pi]^{2}} f(x, y) W(d x) W(d y)+\iint_{[0, \pi]^{2}} f(-x,-y) \overline{W(d x) W(d y)} \\
& +\iint_{[0, \pi]^{2}} f(x,-y) W(d x) \overline{W(d y)}+\iint_{[0, \pi]^{2}} f(-x, y) \overline{W(d x)} W(d y) .
\end{aligned}
$$

When squaring $I_{2}(f)$ and taking its expectation, by the second Itô rule above, the only terms that remain are those for which a product of the form $W(d x) W(d x)$ or $W(d y) W(d y)$ does not appear. Thus we get only two terms left:

$\mathbf{E}\left[I_{2}(f)^{2}\right]=2 \iint_{[0, \pi]^{2}} f(x, y) f(-x,-y) \frac{d x d y}{(2 \pi)^{2}}+2 \iint_{[0, \pi]^{2}} f(x,-y) f(-x, y) \frac{d x d y}{(2 \pi)^{2}}$.

When $f$ satisfies the Hermitian evenness property $f(-x,-y)=\overline{f(x, y)}$, this formula easily yields the isometry property (5.2) announced earlier:

$$
\mathbf{E}\left[I_{2}(f)^{2}\right]=\iint_{[-\pi, \pi]^{2}}|f(x, y)|^{2} \frac{d x d y}{(2 \pi)^{2}}=:\|f\|_{L^{2}\left(\left(S^{1}\right)^{2}\right)}^{2} .
$$

\subsection{Proof of Proposition 1.}

Proof: The constant

$$
\int_{\mathbf{R}}|x|^{1-2 H} \cos (x) L\left(\frac{e \pi}{|x|}\right) d x
$$

is finite because $x \mapsto|x|^{1-2 H} L(e \pi /|x|)$ decreases to 0 as $x \rightarrow \infty$. Thus by (6.4), we get

$$
\frac{\rho(k)}{C_{H, \beta}}=\frac{k^{2 H-2}}{\pi} \int_{0}^{\infty}|x|^{1-2 H} \cos (x) L\left(k \frac{e \pi}{|x|}\right) d x-\frac{k^{2 H-2}}{\pi} \int_{k \pi}^{\infty}|x|^{1-2 H} \cos (x) d x
$$

After integrating by parts, the second term on the right-hand side above can be written as

$$
\begin{aligned}
& \frac{k^{2 H-2}}{\pi} \lim _{N \rightarrow \infty} \int_{k \pi}^{N} x^{1-2 H} \cos (x) d x \\
= & \frac{k^{2 H-2}}{\pi} \lim _{N \rightarrow \infty}\left(N^{1-2 H} \sin N+(2 H-1) \int_{k \pi}^{N} x^{-2 H} \sin (x) d x\right)=\mathcal{O}\left(k^{-1}\right) .
\end{aligned}
$$


Therefore to prove the proposition, it is sufficient to show that

$$
\frac{1}{\pi} \int_{0}^{\infty} x^{1-2 H} \cos (x) L\left(k \frac{e \pi}{x}\right) d x=K_{H} L(k)\left(1+\mathcal{O}\left(\frac{1}{L(k)}\right)\right) .
$$

There is a positive constant $c(\beta)$ such that for $|y|<1$, we have $1-c(\beta)|y| \leq$ $(1+y)^{\beta} \leq 1+c(\beta)|y|$. Thus for $0 \leq y \leq 1$, we can write $(1+y)^{\beta}=1+\mathcal{O}(y)$ where $|\mathcal{O}(y) / y|$ is bounded by $c(\beta)$. We also use $|\cos x| \leq 1$ when $x$ is small. We compute, for any $\varepsilon>0$, for $k$ large,

$$
\begin{aligned}
& \int_{0}^{\infty} x^{1-2 H} \cos (x) L\left(k \frac{e \pi}{x}\right) d x \\
& =\int_{0}^{e \pi / k} x^{1-2 H} \cos (x) L\left(k \frac{e \pi}{x}\right) d x+\int_{e \pi / k}^{\infty}|x|^{1-2 H} \cos (x) \log ^{2 \beta} k\left(1+\frac{\log \left(\frac{e \pi}{|x|}\right)}{\log k}\right)^{2 \beta} d x \\
& =\mathcal{O}\left(\int_{0}^{e \pi / k} x^{1-2 H} L\left(k \frac{e \pi}{x}\right) d x\right)+\int_{e \pi / k}^{\infty}|x|^{1-2 H} \cos (x) \log ^{2 \beta} k\left(1+\mathcal{O}\left(\frac{\log \left(\frac{e \pi}{|x|}\right)}{\log k}\right)\right) d x \\
& =\mathcal{O}\left(k^{2-2 H} \int_{0}^{k^{-2}} y^{1-2 H} \log ^{2 \beta}\left(y^{-1}\right) d y\right)+L(k) \int_{e \pi / k}^{\infty}|x|^{1-2 H} \cos (x) d x \\
& +\mathcal{O}\left(\frac{L(k)}{\log k}\right) \int_{e \pi / k}^{\infty}|x|^{1-2 H} \cos (x) \log \left(\frac{e \pi}{|x|}\right) d x \\
& =\mathcal{O}\left(k^{2-2 H} k^{-2(2-2 H-\varepsilon)}\right)+L(k) \pi K_{H}-L(k) \int_{0}^{e \pi / k}|x|^{1-2 H} \cos (x) d x \\
& +\mathcal{O}\left(\frac{L(k)}{\log k}\right) \int_{0}^{\infty}|x|^{1-2 H} \cos (x) \log \left(\frac{e \pi}{|x|}\right) d x \\
& =\mathcal{O}\left(k^{-(2-2 H)+\varepsilon}\right)+L(k) \pi K_{H}+L(k) \mathcal{O}\left(k^{-(2-2 H)}\right)+\mathcal{O}\left(\frac{L(k)}{\log k}\right)
\end{aligned}
$$

We used the facts that $y \mapsto \int_{0}^{y} x^{1-2 H} d x=\mathcal{O}\left(y^{2-2 H}\right)$ near 0 , that for any $\varepsilon>0$, $y \mapsto \int_{0}^{y} x^{1-2 H} \log ^{\beta}\left(x^{-1}\right) d x=\mathcal{O}\left(y^{2-2 H-\varepsilon}\right)$ near 0 , and that

$\int_{0}^{\infty}|x|^{1-2 H} \cos (x) \log \left(\frac{e \pi}{|x|}\right) d x$ is a converging series. By taking $\varepsilon \in(0,2-2 H)$, this proves the proposition.

7.3. Proof of Proposition 2. Let $\alpha \in(0,1)$. We compute $n v_{n}$ by splitting its series up at the value $k=n^{\alpha}$. We use the notation $\gamma:=2-2 H$ for compactness; note that $1>H>3 / 4$ implies $2 \gamma \in(0,1)$.

To lighten the notation slightly, we write $K_{H, \beta}:=K_{H} C_{H, \beta}$. From Proposition 1 , we compute

$$
\begin{aligned}
& n v_{n}=n+2 K_{H, \beta}^{2}\left[\sum_{k=1}^{\left[n^{\alpha}\right]}+\sum_{k=\left[n^{\alpha}\right]+1}^{n}\right](n-k) k^{-2 \gamma} L^{2}(k)(1+\mathcal{O}(1 / L(k)))^{2} \\
& =n+2 K_{H, \beta}^{2}(1+\mathcal{O}(1)) n^{1+\alpha} \sum_{k=1}^{\left[n^{\alpha}\right]} k^{-2 \gamma} L^{2}(k)(1+\mathcal{O}(1 / L(k)))^{2} \\
& +2 K_{H, \beta}^{2} n^{2-2 \gamma} \frac{1}{n} \sum_{k=\left[n^{\alpha}\right]+1}^{n}\left(1-\frac{k}{n}\right)\left(\frac{k}{n}\right)^{-2 \gamma} L^{2}(k)\left(1+\mathcal{O}\left(1 / L\left(n^{\alpha}\right)\right)\right)^{2}
\end{aligned}
$$


In line (7.3), the term $n$ is negligible in front of the remainder of that line (we already knew this from Corollary 3.3), which is of order at least $n^{1+\alpha} \sum_{k=1}^{\left[n^{\alpha}\right]} k^{-2 \gamma} \asymp$ $n^{1+\alpha+\alpha(1-2 \gamma)}=n^{1+\alpha(1-2 \gamma)}$ and no greater than $n^{1+\alpha(1-2 \gamma)} L^{2}(n)$. Thus the term in line $(7.3)$ is $\mathcal{O}\left(n^{1+\alpha(1-2 \gamma)} L^{2}(n)\right)$. On the other hand, we set up the term in line (7.4) to draw a precise comparison with a Riemann sum; thus modulo the factor $L^{2}(k)\left(1+\mathcal{O}\left(1 / L\left(n^{\alpha}\right)\right)\right)^{2}$ which is smaller than any power, we have an expression which is asymptotically equivalent to a constant times $n^{2-2 \gamma}$. However we find $1+\alpha(1-2 \gamma)<2-2 \gamma \Longleftrightarrow 2 \gamma<1$. Thus the terms in line (7.4) dominate those in line (7.3) by a factor greater than a small power. In other words, we have proved that for some $\varepsilon>0$,

$$
\begin{aligned}
n v_{n}=2 K_{H, \beta}^{2}\left(1+\mathcal{O}\left(1 / L\left(n^{\alpha}\right)\right)\right)^{2} & \left(1+\mathcal{O}\left(n^{-\varepsilon}\right)\right) \\
& \times n^{2-2 \gamma} \frac{1}{n} \sum_{k=\left[n^{\alpha}\right]+1}^{n}\left(1-\frac{k}{n}\right)\left(\frac{k}{n}\right)^{-2 \gamma} L^{2}(k) .
\end{aligned}
$$

Since $L\left(n^{\alpha}\right)=\mathcal{O}(L(n))$ trivially, and $(1+\mathcal{O}(1 / L(n)))^{p}=(1+\mathcal{O}(1 / L(n)))$ for $p>0$, and for any integer $k \geq n^{\alpha}$ we can write

$$
L(k)=\log ^{4 \beta}(n)\left(1+\frac{\log (k / n)}{\log n}\right)^{4 \beta}=L(n)\left(1+\mathcal{O}\left(\frac{1}{\log n}\right)\right),
$$

we get

$$
n v_{n}=2 K_{H, \beta}^{2}\left(1+\mathcal{O}\left(\frac{1}{L(n)}\right)\right) n^{2-2 \gamma} L^{2}(n) \frac{1}{n} \sum_{k=\left[n^{\alpha}\right]+1}^{n}\left(1-\frac{k}{n}\right)\left(\frac{k}{n}\right)^{-2 \gamma}
$$

We must now compute the asymptotics of the series in the last line above. Let $h_{\gamma}(x):=(1-x) x^{-2 \gamma}$ defined on $(0,1]$. We compute $h_{\gamma}^{\prime}(x)=x^{-2 \gamma}(2 \gamma-1-2 \gamma / x)<$ 0 and find $\left|h_{\gamma}^{\prime}(x)\right| \leq 2 x^{-2 \gamma-1}$. We thus have

$$
\begin{aligned}
& \left|\frac{1}{n} \sum_{k=\left[n^{\alpha}\right]+1}^{n} h_{\gamma}\left(\frac{k}{n}\right)-\int_{0}^{1} h_{\gamma}(x) d x\right| \leq \frac{1}{n} \int_{n^{\alpha-1}}^{1} 2 x^{-2 \gamma-1} d x+\int_{0}^{n^{\alpha-1}} x^{-2 \gamma} d x \\
= & \mathcal{O}\left(n^{2 \gamma(1-\alpha)-1}\right)+\mathcal{O}\left(n^{2 \gamma(1-\alpha)-1+\alpha}\right)=\mathcal{O}\left(n^{-(1-\alpha)(1-2 \gamma)}\right) .
\end{aligned}
$$

This proves that for any choice of $\alpha \in(0,1)$

$$
\begin{aligned}
n v_{n} & =2 K_{H, \beta}^{2}\left(1+\mathcal{O}\left(\frac{1}{L(n)}\right)\right) n^{2-2 \gamma} L^{2}(n) \int_{0}^{1} h_{\gamma}(x) d x\left(1+O\left(n^{-(1-\alpha)(1-2 \gamma)}\right)\right) \\
& =2 K_{H, \beta}^{2}\left(\int_{0}^{1} h_{\gamma}(x) d x\right) n^{2-2 \gamma} L^{2}(n)\left(1+\mathcal{O}\left(\frac{1}{L(n)}\right)\right)
\end{aligned}
$$

which is the proposition's claim, given $\gamma=2-2 H$ and the computation

$$
\int_{0}^{1} h_{\gamma}(x) d x=\int_{0}^{1} x^{-2 \gamma} d x+\int_{0}^{1} x^{-2 \gamma+1} d x=\frac{1}{(1-2 \gamma)(2-2 \gamma)} .
$$

\section{Acknowledgements}

The authors thank the anonymous referees for pointing out the mistakes in the initial version. The first author gratefully acknowledges the support for this research from Professor Soledad Torres and the Centro de Investigación y Modelamiento de 
Fenómenos Aleatórios (CIMFAV) of the Universidad de Valparaíso, Chile, as well as from the Ingénieur program at Ecole Polytechnique in Palaiseau, France. The second author's research was partially supported by NSF grant DMS 0907321, and by the CIMFAV.

\section{References}

H. Biermé, A. Bonami and J. R. León. Central limit theorems and quadratic variations in terms of spectral density. Electron. J. Probab. 16, no. 13, 362-395 (2011). MR2774094.

H. Biermé, A. Bonami, I. Nourdin and G. Peccati. Optimal Berry-Esseen rates on the Wiener space: the barrier of third and fourth cumulants. ALEA Lat. Am. J. Probab. Math. Stat. 9 (2), 473-500 (2012). MR3069374.

J.-C. Breton and I. Nourdin. Error bounds on the non-normal approximation of Hermite power variations of fractional Brownian motion. Electron. Commun. Probab. 13, 482-493 (2008). MR2447835.

P. Breuer and P. Major. Central limit theorems for non-linear functionals of gaussian fields. J. Mult. Anal. 13 (3), 362-395 (2011). DOI: 10.1016/0047259X(83)90019-2.

Yu. A. Davydov and G. V. Martynova. Limit behavior of distributions of multiple stochastic integrals. In Statistics and control of random processes (Russian) (Preila, 1987), pages 55-57. "Nauka", Moscow (1989).

R. L. Dobrushin. Gaussian and their subordinated self-similar random generalized fields. Ann. Probab. 7 (1), 1-28 (1979). MR515810.

R. L. Dobrushin and P. Major. Non-central limit theorems for nonlinear functionals of Gaussian fields. Z. Wahrsch. Verw. Gebiete 50 (1), 27-52 (1979). MR550122.

I. Nourdin and G. Peccati. Stein's method on Wiener chaos. Probab. Theory Related Fields 145 (1-2), 75-118 (2009). MR2520122.

I. Nourdin and G. Peccati. Normal approximations with Malliavin calculus, volume 192 of Cambridge Tracts in Mathematics. Cambridge University Press, Cambridge (2012). ISBN 978-1-107-01777-1. From Stein's method to universality. MR2962301.

I. Nourdin and G. Peccati. The optimal fourth moment theorem. Proc. Amer. Math. Soc. 143 (7), 3123-3133 (2015). MR3336636.

I. Nourdin, G. Peccati and G. Reinert. Invariance principles for homogeneous sums: universality of Gaussian Wiener chaos. Ann. Probab. 38 (5), 1947-1985 (2010). MR2722791.

I. Nourdin and G. Poly. Convergence in law in the second Wiener/Wigner chaos. Electron. Commun. Probab. 17, no. 36, 12 (2012). MR2970700.

D. Nualart and G. Peccati. Central limit theorems for sequences of multiple stochastic integrals. Ann. Probab. 33 (1), 177-193 (2005). MR2118863.

E. M. Stein and R. Shakarchi. Fourier analysis, volume 1 of Princeton Lectures in Analysis. Princeton University Press, Princeton, NJ (2003). ISBN 0-691-11384-X. An introduction. MR1970295.

M. S. Taqqu. Convergence of integrated processes of arbitrary Hermite rank. $Z$. Wahrsch. Verw. Gebiete 50 (1), 53-83 (1979). MR550123.

C. A. Tudor and F. G. Viens. Variations and estimators for self-similarity parameters via Malliavin calculus. Ann. Probab. 37 (6), 2093-2134 (2009). MR2573552. 